TRANSACTIONS OF THE

AMERICAN MATHEMATICAL SOCIETY

Volume 359, Number 6, June 2007, Pages 2625-2650

S 0002-9947(07)03948-7

Article electronically published on January 25, 2007

\title{
DEFORMED PREPROJECTIVE ALGEBRAS OF GENERALIZED DYNKIN TYPE
}

\author{
JERZY BIAŁKOWSKI, KARIN ERDMANN, AND ANDRZEJ SKOWROŃSKI
}

\begin{abstract}
We introduce the class of deformed preprojective algebras of generalized Dynkin graphs $\mathbb{A}_{n}(n \geq 1), \mathbb{D}_{n}(n \geq 4), \mathbb{E}_{6}, \mathbb{E}_{7}, \mathbb{E}_{8}$ and $\mathbb{L}_{n}(n \geq 1)$ and prove that it coincides with the class of all basic connected finite-dimensional self-injective algebras for which the inverse Nakayama shift $\nu^{-1} S$ of every non-projective simple module $S$ is isomorphic to its third syzygy $\Omega^{3} S$.
\end{abstract}

\section{INTRODUCTION AND THE MAIN RESULTS}

We fix an algebraically closed field $K$. By an algebra we mean a finite-dimensional $K$-algebra (associative, with identity) which we moreover assume to be basic and connected. Any such algebra $A$ can be written as a bound quiver algebra, that is $A \cong K Q / I$, where $Q=Q_{A}$ is the Gabriel quiver of $A$ and $I$ is an admissible ideal in the path algebra $K Q$ of $Q$.

Preprojective algebras were introduced by Gelfand and Ponomarev GP (and implicitly in the work of Riedtmann [Rie] to study the preprojective representations of finite quivers without oriented cycles (see also BGL, DR1, DR2, Rin1, Rin2 for generalizations and properties). Subsequently it was discovered that preprojective algebras occur naturally in very diverse contexts. For example, they have been used by Kronheimer $[\mathrm{K}]$ to deal with problems in differential geometry, also by Lusztig [L1, L2 in his perverse sheaf approach to quantum groups, and by Crawley-Boevey and Holland $[\mathrm{CBH}]$ to study non-commutative deformations of Kleinian singularities.

Traditionally preprojective algebras are defined as follows. Let $Q$ be a finite connected quiver with a fixed point free involution $a \rightarrow \bar{a}$ on the arrows such that $i \bar{a}=t a$ and $t \bar{a}=i a$, where $i b$ and $t b$ denote the starting and ending points of an arrow $b$. The preprojective algebra associated to this quiver is the bound quiver algebra $K Q / I$ where $I$ is the ideal in the path algebra $K Q$ of $Q$ generated by the relations of the form

$$
\sum_{a, i a=v} a \bar{a} \quad(v \text { a vertex of } Q) .
$$

The associated graph $\Delta=\Delta_{Q}$ of the preprojective algebra is obtained by replacing each pair of arrows $\{a, \bar{a}\}$ by an undirected edge. This determines the preprojective algebra completely, and hence it is denoted by $P(\Delta)$. It is well-known that the

Received by the editors September 13, 2004 and, in revised form, February 15, 2005.

2000 Mathematics Subject Classification. Primary 16D50, 16E30, 16E40, 16G20, 16G60, 16P10, 18G99.

The first and third named authors gratefully acknowledge support from the Polish Scientific Grant KBN No. 1 P03A 01827.

(C)2007 American Mathematical Society Reverts to public domain 28 years from publication 
algebra $P(\Delta)$ is finite-dimensional if and only if $\Delta$ is one of the Dynkin graphs $\mathbb{A}_{n}$, $\mathbb{D}_{n}, \mathbb{E}_{6}, \mathbb{E}_{7}$, or $\mathbb{E}_{8}$ (see [DR1], GP], Rie]). Moreover, in this case, the algebra $P(\Delta)$ is self-injective. The module category of a finite-dimensional preprojective algebra $P(\Delta)$ is known to be quite exceptional, namely all non-projective indecomposable finite-dimensional modules have $\Omega$-period at most six, where $\Omega$ is Heller's syzygy operator which assigns to any module $M$ the kernel of its projective cover (see AR2, [B], ES1, ES2, [S]). We also note that the preprojective algebras $P(\Delta)$ of Dynkin graphs $\Delta$ other than $\mathbb{A}_{n}(1 \leq n \leq 5)$ and $\mathbb{D}_{4}$ are of wild representation type (see [BS1, [BS2, [DR2]), and these first give natural examples of wild algebras whose stable Auslander-Reiten quiver consists only of tubes.

One would like to know which self-injective algebras have similar periodicity properties. Surprisingly these can be described very explicitly. We will now introduce the algebras which are of central interest in this paper. Let $Q$ be a finite connected quiver with an involution $a \rightarrow \bar{a}$ of the arrows such that $i \bar{a}=t a$ and $t \bar{a}=i a$, and assume that the involution fixes all loops of $Q$ and acts freely on the remaining arrows of $Q$. Then again the preprojective algebra, defined as above, is uniquely determined by the graph $\Delta=\Delta_{Q}$ obtained from $Q$ by replacing each pair $\{a, \bar{a}\}$ of arrows by an undirected edge, and we denote it by $P(\Delta)$. Observe now that the numbers of loops in $Q$ and $\Delta_{Q}$ are the same. Then the algebra $P(\Delta)$ is finite-dimensional if and only if $\Delta$ is one of Dynkin graphs $\mathbb{A}_{n}, \mathbb{D}_{n}, \mathbb{E}_{6}, \mathbb{E}_{7}, \mathbb{E}_{8}$, or else is a graph of the form

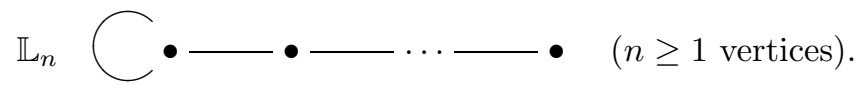

Following [HPR1], the graphs $\mathbb{A}_{n}, \mathbb{D}_{n}, \mathbb{E}_{6}, \mathbb{E}_{7}, \mathbb{E}_{8}$ and $\mathbb{L}_{n}$ are called generalized Dynkin graphs. They coincide with the graphs associated to the indecomposable finite symmetric generalized Cartan matrices having subadditive functions which are not additive (see also HPR2, or see section 4). We will show (in Section 2) that $P\left(\mathbb{L}_{n}\right)$ is self-injective and that all its non-projective indecomposable finitedimensional modules have $\Omega$-period at most 3 . In this paper, the preprojective algebras $P(\Delta)$ with $\Delta \in\left\{\mathbb{A}_{n}, \mathbb{D}_{n}, \mathbb{E}_{6}, \mathbb{E}_{7}, \mathbb{E}_{8}, \mathbb{L}_{n}\right\}$ are said to be the preprojective algebras of generalized Dynkin type. These self-injective algebras also occur naturally in the study of Cohen-Macaulay modules and lattices over orders. Namely, it is well-known that if $R$ is a complete local noetherian isolated two-dimensional hypersurface singularity of finite Cohen-Macaulay type such that $K[[X, Y]] \subset R$ and $R$ a finitely generated free $K[[X, Y]]$-module, then the stable Auslander algebra $\underline{\operatorname{End}}_{R}(M)$ of the direct sum $M$ of one copy of each indecomposable (maximal) Cohen-Macaulay $R$-module is isomorphic to the preprojective algebra $P(\Delta)$, where $\Delta$ is the Dynkin graph associated to $R$ (see AR1, AR21). This result has been extended in AR1 to the non-commutative two-dimensional case, namely to all orders of finite lattice type over complete regular local two-dimensional rings $T$ and with the identity Auslander-Reiten translation $\tau$, where the stable Auslander algebra is isomorphic to a preprojective algebra of generalized Dynkin type. The computations in AR1] (based on the Artin's classification [A]) show that in the non-commutative two-dimensional case the preprojective algebras $P\left(\mathbb{L}_{n}\right)$ actually do occur.

The preprojective algebras of Dynkin type have the following distinguished homological property: the inverse Nakayama shift $\nu^{-1} M$ of every non-projective indecomposable finite-dimensional module $M$ is isomorphic to its third syzygy $\Omega^{3} M$ 
(see [AR2, (3.1)-(3.3)]). Recall that, for a self-injective algebra $\Lambda$, the Nakayama automorphism $\nu$ of the category $\bmod \Lambda$ of finite-dimensional right $\Lambda$-modules is the functor $\nu=D \operatorname{Hom}_{\Lambda}(-, \Lambda)$, where $D=\operatorname{Hom}_{K}(-, K)$ is the standard duality on $\bmod \Lambda$ ARS. Its inverse $\nu^{-1}=\operatorname{Hom}_{\Lambda^{\mathrm{op}}}(-, \Lambda) D$ assigns to any simple $\Lambda$-module $S$ the socle of the projective cover $P(S)$ of $S$. Alternatively, there is an algebra automorphism such that $\nu^{-1} S$ is isomorphic to the twist of $S$ with this automorphism, for all simple $\Lambda$-modules $S$.

The main objective of the paper is to give a complete description of all basic, connected, finite-dimensional, self-injective algebras $\Lambda$ with the property that all its simple right modules $S$ satisfy $\Omega^{3} S \cong \nu^{-1} S$. To each generalized Dynkin graph $\Delta$ we associate a finite-dimensional self-injective algebra $R(\Delta)$, isomorphic to the local algebra $e P(\Delta) e$ of the preprojective algebra $P(\Delta)$ at the exceptional primitive idempotent $e$ of $P(\Delta)$ (see Section 3 for details). Then, for each element $f$ which belongs to the square of the radical of $R(\Delta)$, we define the deformed preprojective algebra $P^{f}(\Delta)$ of type $\Delta$, which degenerates to $P(\Delta)$ in the corresponding affine variety of algebras. We will establish basic homological properties:

Theorem 1.1. Let $\Lambda=P^{f}(\Delta)$ be a deformed preprojective algebra of a generalized Dynkin type $\Delta$. Then the following hold:

(i) $\Lambda$ is self-injective and has the same Cartan matix as $P(\Delta)$, in particular $\operatorname{dim}_{K} \Lambda=\operatorname{dim}_{K} P(\Delta)$.

(ii) There are Nakayama automorphisms $\eta^{f}$ and $\eta$ of $P^{f}(\Delta)$ and $P(\Delta)$ which induce the same automorphism modulo the square of the radical.

(iii) $\Omega_{\Lambda^{e}}^{3}(\Lambda) \cong{ }_{1} \Lambda_{\widetilde{\nu}}$, as a $\Lambda$ - $\Lambda$-bimodule, for an automorphism $\widetilde{\nu}$ of $\Lambda$ of finite order.

(iv) There is a positive integer $m=m_{\Lambda}$ such that $\Omega^{3 m} M \cong M$ for any nonprojective indecomposable finite-dimensional right $\Lambda$-module $M$.

(v) $\Omega^{3} S \cong \nu^{-1} S$ for any simple right $\Lambda$-module $S$.

The following theorem is the main result of the paper.

Theorem 1.2. Let $\Lambda$ be a basic, connected, finite-dimensional, self-injective algebra over an algebraically closed field $K$. Then the following statements are equivalent:

(i) $\Lambda$ is isomorphic to a deformed preprojective algebra $P^{f}(\Delta)$ of a generalized Dynkin type $\Delta$.

(ii) $\Omega^{3} S \cong \nu^{-1} S$ for any non-projective simple right $\Lambda$-module $S$.

We do not know when, for a deformed preprojective algebra $\Lambda=P^{f}(\Delta)$ of a generalized Dynkin type $\Delta$, we have $\Omega^{3} M \cong \nu^{-1} M$ for all non-projective indecomposable finite-dimensional right $\Lambda$-modules $M$. It is the case for all preprojective algebras of generalized Dynkin type (Corollary 2.6) but also for some deformed preprojective algebras of generalized Dynkin type (Example 3.6).

Our final result shows that there exist deformed preprojective algebras $P^{f}(\Delta)$ of generalized Dynkin type $\Delta$ which are not isomorphic to the ordinary preprojective algebras $P(\Delta)$.

Theorem 1.3. Let $K$ be an algebraically closed field of characteristic 2. Then for any generalized Dynkin graph $\Delta$ other than $\mathbb{A}_{n}$ and $\mathbb{L}_{1}$, there exists a deformed preprojective algebra $P^{f}(\Delta)$ over $K$ which is not isomorphic to the preprojective algebra $P(\Delta)$. 
A complete classification of the isomorphism classes of the deformed preprojective algebras of generalized Dynkin type is a rather complicated combinatorial problem, to which we hope to return in a forthcoming paper.

The paper is organized as follows. In Section 2 we fix notations and extend the known homological properties of the preprojective algebras of Dynkin type to the preprojective algebras of generalized Dynkin type. In Section 3 we introduce the deformed preprojective algebras of generalized Dynkin type and prove Theorem 1.1 . In Section 4 we describe the Gabriel quivers, Cartan matrices and Nakayama permutations of self-injective algebras satisfying the considered homological condition on simples. Sections 5 and 6 are devoted to the proofs of Theorems 1.2 and 1.3 respectively.

\section{Preprojective algebras of Generalized Dynkin type}

In this section we extend the known periodicity theorem on the preprojective algebras of Dynkin type to the preprojective algebras of generalized Dynkin type.

Let $\Delta$ be a generalized Dynkin graph and $\Lambda=P(\Delta)$ the associated preprojective algebra. We fix the following labelling of the Gabriel quiver $Q_{\Lambda}$ of $\Lambda$ (see [ES1, ES2 ]):

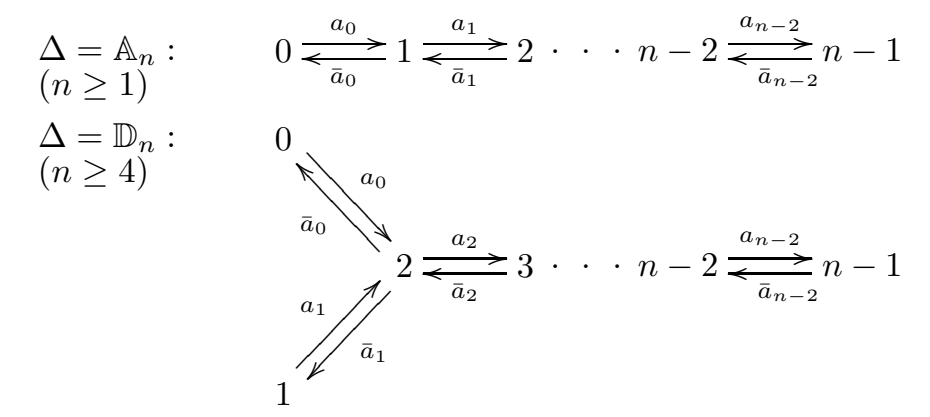

$$
\begin{aligned}
& \Delta=\mathbb{E}_{n}: \\
& (n=6,7,8)
\end{aligned}
$$

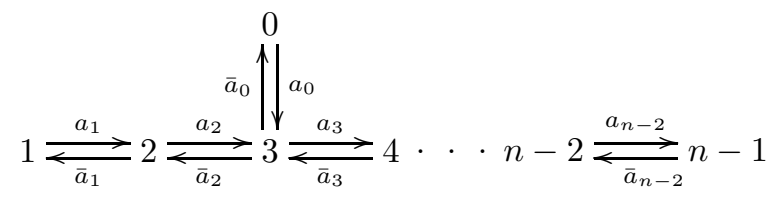

$$
\begin{aligned}
& \underset{(n \geq 1)}{\Delta=\mathbb{L}_{n}:} \quad \varepsilon=\bar{\varepsilon} \bigodot 0 \underset{\bar{a}_{0}}{\stackrel{a_{0}}{\rightleftarrows}} 1 \underset{\bar{a}_{1}}{\stackrel{a_{1}}{\rightleftarrows}} 2 \cdots n-2 \underset{\bar{a}_{n-2}}{\stackrel{a_{n-2}}{\rightleftarrows}} n-1 .
\end{aligned}
$$

We have the following straightforward extension of [DR2, (2.1) and (6.2)] to the arbitrary generalized Dynkin graph case.

Proposition 2.1. The algebra $P(\Delta)$ is finite-dimensional self-injective. Its Nakayama permutation $\nu$ is the identity for $\Delta=\mathbb{A}_{1}, \mathbb{D}_{n}$ ( $n$ even), $\mathbb{E}_{7}, \mathbb{E}_{8}, \mathbb{L}_{n}$, and of order 2 for $\Delta=\mathbb{A}_{n}(n \geq 2), \mathbb{D}_{n}$ ( $n$ odd $), \mathbb{E}_{6}$.

It is proved in $[\mathrm{S}$ that, for $\Delta$ a Dynkin graph, the $\Lambda$ - $\Lambda$-bimodule $\Lambda$ has a periodic minimal projective resolution of periodicity at most 6 . The construction of such resolution can be extended to the case $\Delta=\mathbb{L}_{n}$. Define projective $\Lambda$ - $\Lambda$-bimodules 
(equivalently modules over $\Lambda^{e}=\Lambda \otimes \Lambda^{o p}$ )

$$
\begin{gathered}
P_{0}=P_{2}=\bigoplus_{i} \Lambda\left(e_{i} \otimes e_{i}\right) \Lambda \quad\left(\text { sum over all vertices of } Q_{\Lambda}\right), \\
P_{1}=\bigoplus_{a} \Lambda\left(e_{i a} \otimes e_{t a}\right) \Lambda \quad\left(\text { sum over all arrows of } Q_{\Lambda}\right),
\end{gathered}
$$

and define homomorphisms $\delta: P_{1} \rightarrow P_{0}$ and $R: P_{2} \rightarrow P_{1}$ by

$$
\begin{gathered}
\delta\left(e_{i a} \otimes e_{t a}\right)=a \otimes e_{t a}-e_{i a} \otimes a=: x_{a}, \\
R\left(e_{i} \otimes e_{i}\right)=\sum_{i a=i} e_{i a} \otimes \bar{a}+a \otimes e_{t \bar{a}}=: \sigma_{i}
\end{gathered}
$$

where $\otimes$ means $\otimes_{K}$ (this should not cause confusion). Moreover, let $u: P_{0} \rightarrow \Lambda$ be the multiplication map. Then we have an exact sequence of $\Lambda$ - $\Lambda$-bimodules

$$
0 \longrightarrow \Omega_{\Lambda^{e}}^{3}(\Lambda) \longrightarrow P_{2} \stackrel{R}{\longrightarrow} P_{1} \stackrel{\delta}{\longrightarrow} P_{0} \stackrel{u}{\longrightarrow} \Lambda \longrightarrow 0 .
$$

Theorem 2.2. Let $\Lambda=P(\Delta)$ for a generalized Dynkin graph $\Delta$. Then the third syzygy $\Omega_{\Lambda^{e}}^{3}(\Lambda)$ is isomorphic as a $\Lambda$ - $\Lambda$-bimodule to ${ }_{1} \Lambda_{\widetilde{\nu}}$, the bimodule with the right action twisted by an automorphism $\widetilde{\nu}$ of $\Lambda$. Moreover, the $\Lambda$ - $\Lambda$-bimodules ${ }_{1} \Lambda_{\widetilde{\nu}^{2}}$ and $\Lambda$ are isomorphic.

For $\Delta$ a Dynkin graph, this theorem has been proved in [S] (see also [ES2] for a correction). It remains to deal with $\Delta=\mathbb{L}_{n}$, which is done by the following proposition.

Proposition 2.3. Let $\Lambda=P\left(\mathbb{L}_{n}\right), n \geq 1$. There is a minimal projective bimodule resolution

$$
0 \longrightarrow{ }_{1} \Lambda_{\sigma} \longrightarrow P_{2} \stackrel{R}{\longrightarrow} P_{1} \stackrel{\delta}{\longrightarrow} P_{0} \stackrel{u}{\longrightarrow} \Lambda \longrightarrow 0
$$

where $\sigma=-i d_{\Lambda}$.

Before proving this, we recall the general construction of $\Omega^{3}(\Lambda)$ as a $\Lambda$ - $\Lambda$ bimodule given in ES2] here $\Lambda$ is an arbitrary self-injective basic algebra. Since $\Lambda$ is self-injective, there is a non-singular bilinear form $(-,-): \Lambda \times \Lambda \rightarrow K$ such that $(x y, z)=(x, y z)$ for $x, y, z \in \Lambda$. This is associated with a 'Nakayama automorphism' of $\Lambda$ which we call $\eta$, characterized by $(x, y)=(y, \eta(x))$ for $x, y \in \Lambda$. (Note that the notation in ES2 is different.) Thus $\eta\left(e_{i}\right)=e_{\nu^{-1}(i)}$, where $\nu$ is the Nakayama permutation of $\Lambda$ introduced earlier. Let $e_{i}$ denote the primitive idempotent corresponding to vertex $i$ of $Q$ and let $\omega_{i}$ be a fixed non-zero element in the socle of $e_{i} \Lambda$.

Fix a $K$-basis $\mathcal{B}$ of $\Lambda$ such that each $v \in \mathcal{B}$ belongs to $e_{i} \Lambda e_{j}$ for some $i, j$, and moreover assume that the basis $\mathcal{B}$ contains the primitive idempotents $e_{i}$, the arrows and the $\omega_{i}$. Then one can take the non-singular bilinear form $(-,-)$ explicitly as follows. For $x, y \in \mathcal{B}$ and $x=e_{i} x$, set

$$
(x, y):=\text { the coefficient of } \omega_{i} \text { in } x y
$$

when $x y$ is expressed in terms of $\mathcal{B}$. Given $\mathcal{B}$, there is a dual $K$-basis $\mathcal{B}^{*}=\left\{b^{*}, b \in\right.$ $\mathcal{B}\}$ such that $\left(b, c^{*}\right)=\delta_{b c}$ for $b, c \in \mathcal{B}$.

For preprojective algebras of Dynkin type, the defining relations show that there is such a basis which is almost multiplicative, that is, the product of two basis elements is zero or \pm a basis element. Take such a basis; then $v \in e_{i} \mathcal{B} e_{j}$ if and only 
if $\pm v^{*} \in e_{j} \mathcal{B}^{*} e_{\nu^{-1}(i)}$. Following [ES1, (2.2)] we define elements $\xi_{i} \in \bigoplus_{i} \Lambda\left(e_{i} \otimes e_{i}\right) \Lambda$ by the formula

$$
\xi_{i}:=\sum_{x \in e_{i} \mathcal{B}}(-1)^{\operatorname{deg}(x)} x \otimes x^{*} .
$$

Proof of Proposition 2.3. Let $\Lambda=P\left(\mathbb{L}_{n}\right)$. It follows from [H] (1.5)] that also for $\Lambda=P\left(\mathbb{L}_{n}\right)$,

$$
P_{0}=\bigoplus_{i} \Lambda\left(e_{i} \otimes e_{i}\right) \Lambda, \quad P_{1}=\bigoplus_{a} \Lambda\left(e_{i a} \otimes e_{t a}\right) \Lambda, \quad P_{2}=P_{0}
$$

are the projective $\Lambda^{e}$-modules occurring in a minimal projective bimodule resolution of $\Lambda$ in degrees 0,1 and 2, respectively. Clearly, the multiplication map $u: P_{0} \rightarrow \Lambda$ is surjective. Further, the elements $x_{a}$ lie in the kernel of $u$ but not in the radical of $\operatorname{Ker}(u)$, and hence $\delta: P_{1} \rightarrow \operatorname{Ker}(u)$ is a projective cover. It is easy to check that $\delta \circ R=0$ and hence $\operatorname{Im}(R) \subseteq \operatorname{Ker}(\delta)$. In fact, the elements $\sigma_{i}$ do not lie in the radical of $\operatorname{Ker}(\delta)$ and hence $\operatorname{Ker}(\delta)=\operatorname{Im}(R)$. Finally, by [ES1, (2.3)] we have $R\left(\xi_{i}\right)=0$. Let $N$ be the subbimodule of $P_{2}$ generated by the elements $\xi_{i}$. Then invoking [ES1, (2.3)] we conclude that $N=\operatorname{Ker}(R)=\Omega_{\Lambda^{e}}^{3}(\Lambda)$.

We will now identify $N$ as a twisted bimodule. It follows from [ES1, (2.3)] that $N$ is isomorphic to $\Lambda$ as a left module and as a right module. Applying arguments from GSS, (1.4)] we conclude that $N$ is a twisted bimodule by an automorphism of $\Lambda$ which fixes the primitive idempotents $e_{i}$. In order to identify the action on the arrows we only need to compare $a \xi_{i}$ and $\xi_{j} a$ where $a$ is an arrow with $a=e_{i} a e_{j}$. We want to show that $a \xi_{i}=-\xi_{j} a$. We need the following lemma.

Lemma 2.4. For $m$ in the basis $\mathcal{B}$ and an arrow a we have

$$
a(m a)^{*}=m^{*} \quad \text { and } \quad(a m)^{*} a=m^{*} .
$$

Proof. The first equality is proved in [ES1, (2.3.1)]. For the second equality, let $a$ be an arrow from $i$ to $j$ such that $m=e_{j} m$. Then $(a m)(a m)^{*}=\omega_{i}$. By the rotation convention we have $m(a m)^{*} a=\omega_{j}$, and hence $(a m)^{*} a=m^{*}$.

We now claim that $\varepsilon \xi_{0}=-\xi_{0} \varepsilon$. That is, we want to show that $(\mathrm{I})=-(\mathrm{II})$, where

(I) $\varepsilon \xi_{0}=\sum_{x}(-1)^{\operatorname{deg}(x)} \varepsilon x \otimes x^{*}$,

(II) $\xi_{0} \varepsilon=\sum_{x}(-1)^{\operatorname{deg}(x)} x \otimes x^{*} \varepsilon$.

If in (I) $x=\omega_{0}$, then $\varepsilon x=0$, and this matches up with the term for $x=e_{0}$ in (II). Now let $x \neq \omega_{0}$ be an element of $\mathcal{B}$. Then $\pm \varepsilon x \in \mathcal{B}$. We need to show that the following elements add up to zero:

$$
(-1)^{\operatorname{deg}(x)} \varepsilon x \otimes x^{*} \quad \text { and } \quad(-1)^{\operatorname{deg}(\varepsilon x)}( \pm \varepsilon x) \otimes( \pm \varepsilon x)^{*} \varepsilon .
$$

Applying Lemma 2.4 to $m= \pm x$ and $a=\varepsilon$ we get that the first term is the minus of the second term. The same arguments work exactly for all other arrows. Therefore, the $\Lambda$ - $\Lambda$-bimodules $N$ and ${ }_{1} \Lambda_{\sigma}$, with $\sigma=-i d_{\Lambda}$, are isomorphic. This finishes the proof.

Corollary 2.5. Let $\Lambda=P\left(\mathbb{L}_{n}\right), n \geq 1$. Then for any non-projective indecomposable finite-dimensional right $\Lambda$-module $M$ we have $\Omega_{\Lambda}^{3}(M) \cong M$.

Proof. Applying Proposition 2.3 we obtain $\Omega_{\Lambda}^{3}(M) \cong M \otimes_{\Lambda} \Omega_{\Lambda^{e}}^{3}(\Lambda) \cong M \otimes_{\Lambda 1} \Lambda_{\sigma} \cong$ $M$ since $\sigma$ is a multiplication by a non-zero scalar. 
The following corollary is a direct consequence of [AR2, (3.1)-(3.3)], Theorem 2.2 and Corollary 2.5

Corollary 2.6. Let $\Lambda=P(\Delta)$ be a preprojective algebra of a generalized Dynkin type $\Delta$. Then for any non-projective indecomposable finite-dimensional right $\Lambda$ module $M$ we have $\Omega^{3}(M) \cong \nu^{-1} M$ and $\Omega^{6}(M) \cong M$.

\section{Deformed preprojective algebras of generalized Dynkin type}

In this section we introduce the deformed preprojective algebras of generalized Dynkin type and prove Theorem 1.1 .

Let $\Delta$ be a generalized Dynkin graph. We define an associated algebra $R(\Delta)$ as follows:

$$
\begin{aligned}
& R\left(\mathbb{A}_{n}\right)=K, \\
& R\left(\mathbb{D}_{n}\right)=K\langle x, y\rangle /\left(x^{2}, y^{2},(x+y)^{n-2}\right), \\
& R\left(\mathbb{E}_{6}\right)=K\langle x, y\rangle /\left(x^{2}, y^{3},(x+y)^{3}\right), \\
& R\left(\mathbb{E}_{7}\right)=K\langle x, y\rangle /\left(x^{2}, y^{3},(x+y)^{4}\right), \\
& R\left(\mathbb{E}_{8}\right)=K\langle x, y\rangle /\left(x^{2}, y^{3},(x+y)^{5}\right), \\
& R\left(\mathbb{L}_{n}\right)=K[x] /\left(x^{2 n}\right) .
\end{aligned}
$$

Further, we choose the exceptional vertex in the Gabriel quiver $Q_{P(\Delta)}$ of the preprojective algebra $P(\Delta)$ (in the notation of Section 2) as: $0,2,3,3,3$ and 0 if $\Delta=\mathbb{A}_{n}, \mathbb{D}_{n}, \mathbb{E}_{6}, \mathbb{E}_{7}, \mathbb{E}_{8}$ and $\mathbb{L}_{n}$, respectively. Moreover, we denote by $e$ the primitive idempotent of $P(\Delta)$ associated to the chosen exceptional vertex of $Q_{P(\Delta)}$. Then a simple check shows that the following lemma holds.

Lemma 3.1. The algebra $R(\Delta)$ is isomorphic to eP( $\Delta)$ e. In particular, $R(\Delta)$ is local, finite-dimensional and self-injective.

An element $f$ from the square $\operatorname{rad}^{2} R(\Delta)$ of the radical of $R(\Delta)$ is said to be admissible. Note that $f=0$ is the unique admissible element of $R\left(\mathbb{A}_{n}\right)=K$. For an admissible element $f$ of $R(\Delta)$, the deformed preprojective algebra $P^{f}(\Delta)$ of type $\Delta$, with respect to $f$, is defined to be as the bound quiver algebra $K Q / I^{f}$, where $Q=Q_{P(\Delta)}$ and $I^{f}$ is the ideal in the path algebra $K Q$ of $Q$ generated by the relations of the form

$$
\sum_{a, i a=v} a \bar{a}, \quad \text { if } v \text { is an ordinary vertex of } Q
$$

and the relations:

$$
\begin{array}{ll}
a_{0} \bar{a}_{0}, & \Delta=\mathbb{A}_{n}, \\
\bar{a}_{0} a_{0}+\bar{a}_{1} a_{1}+a_{2} \bar{a}_{2}+f\left(\bar{a}_{0} a_{0}, \bar{a}_{1} a_{1}\right),\left(\bar{a}_{0} a_{0}+\bar{a}_{1} a_{1}\right)^{n-2}, & \Delta=\mathbb{D}_{n}, \\
\bar{a}_{0} a_{0}+\bar{a}_{2} a_{2}+a_{3} \bar{a}_{3}+f\left(\bar{a}_{0} a_{0}, \bar{a}_{2} a_{2}\right),\left(\bar{a}_{0} a_{0}+\bar{a}_{2} a_{2}\right)^{n-3}, & \Delta=\mathbb{E}_{n}(n=6,7,8), \\
\varepsilon^{2}+a_{0} \bar{a}_{0}+\varepsilon f(\varepsilon), \varepsilon^{2 n}, & \Delta=\mathbb{L}_{n} .
\end{array}
$$

That is, $P^{f}(\Delta)$ is obtained from $P(\Delta)$ by deforming the relation at the exceptional vertex of $Q$, and $P^{f}(\Delta)=P(\Delta)$ if $f=0$. The following shows that $P^{f}(\Delta)$ is a deformation of $P(\Delta)$.

Lemma 3.2. Let $P^{f}(\Delta)$ be a deformed preprojective algebra of a generalized Dynkin type $\Delta$. Then $P^{f}(\Delta)$ is self-injective with the same Cartan matrix as $P(\Delta)$ and $\operatorname{dim}_{K} P^{f}(\Delta)=\operatorname{dim}_{K} P(\Delta)$. 
Proof. We have $P^{f}(\Delta)=K Q / I^{f}$ and $P(\Delta)=K Q / I$, where $Q=Q_{P(\Delta)}$. Further, the ideals $I^{f}$ and $I$ differ only by one relation at the exceptional vertex, and the difference of these two relations belongs to the ideal of $K Q$ generated by all paths in $Q$ of length $\geq 3$.

Denote by $e_{i}$ the primitive idempotent of $P^{f}(\Delta)$ (respectively, $P(\Delta)$ ) associated to a vertex $i$ of $Q$. Also denote by $e$ the idempotent associated to the exceptional vertex. Observe first that $e P^{f}(\Delta) e$, as an algebra, is isomorphic to $R(\Delta)$. One checks that within this algebra, the relation in which $f$ occurs is redundant. In particular it is finite-dimensional self-injective and of the same dimension as $e P(\Delta) e$. For any other idempotent $e_{i}$, the algebra $e_{i} P^{f}(\Delta) e_{i}$ can be obtained from $e P^{f}(\Delta) e$ by multiplying on both sides with the shortest paths between $e$ and $e_{i}$, and one checks that it is also finite-dimensional self-injective and of the right dimension. More generally, for each vertex $i$ of $Q$ and an integer $m \geq 0$, we have canonical isomorphisms of $K$-vector spaces

$$
e_{i} \operatorname{rad}\left(P^{f}(\Delta)\right)^{m} / e_{i} \operatorname{rad}\left(P^{f}(\Delta)\right)^{m+1} \stackrel{\sim}{\longrightarrow} e_{i}(\operatorname{rad} P(\Delta))^{m} / e_{i}(\operatorname{rad} P(\Delta))^{m+1} .
$$

In fact, if $u_{1}, \ldots, u_{d}$ are paths in $Q$ with source $i$ such that the cosets $u_{j}+$ $e_{i}(\operatorname{rad} P(\Delta))^{m+1}, 1 \leq j \leq d$, form a $K$-basis of $e_{i}(\operatorname{rad} P(\Delta))^{m} / e_{i}(\operatorname{rad} P(\Delta))^{m+1}$, then the cosets $u_{j}+e_{i}\left(\operatorname{rad} P^{f}(\Delta)\right)^{m+1}$ form a basis of $e_{i}\left(\operatorname{rad} P^{f}(\Delta)\right)^{m} /$ $e_{i}\left(\operatorname{rad} P^{f}(\Delta)\right)^{m+1}$. Therefore, $\operatorname{dim}_{K} e_{i} P^{f}(\Delta)=\operatorname{dim}_{K} e_{i} P(\Delta)$ for any vertex $i$ of $Q$, and hence we have $\operatorname{dim}_{K} P^{f}(\Delta)=\operatorname{dim}_{K} P(\Delta)<\infty$. Moreover, for any vertex $i$ of $Q$, the indecomposable projective modules $e_{i} P^{f}(\Delta)$ and $e_{i} P(\Delta)$ have the same Loewy length and the same composition factors. In particular, the modules $e_{i} P^{f}(\Delta)$ have simple socles. Then by a result due to Nakayama (see [Y, (2.2.1)]) $P^{f}(\Delta)$ is self-injective and has the same Nakayama permutation as $P(\Delta)$.

We now compare the Nakayama automorphisms $\eta^{f}$ and $\eta$ of $P^{f}(\Delta)$ and $P(\Delta)$ as defined before Proposition 2.3. Due to the non-homogeneous deformed relation, the automorphisms $\eta^{f}$ and $\eta$ are not always the same (we do not know when they might just differ by an inner automorphism). However, let $\bar{\eta}^{f}$ and $\bar{\eta}$ be the automorphisms induced on the the quotient modulo the square of the radical. Then we have

Lemma 3.3. Let $P^{f}(\Delta)$ be a deformed preprojective algebra of a generalized Dynkin type $\Delta$. Then there are Nakayama automorphisms $\eta^{f}$ and $\eta$ of $P^{f}(\Delta)$ and $P(\Delta)$ which induce the same automorphisms on the quotients modulo the square of the radical.

Proof. We use the notation and the setup as described after Proposition 2.3, and we modify the arguments in ES2. Let $\nu$ be the Nakayama permutation; then for any arrow $a$ we have

$$
\bar{\eta}(a)=c_{a}^{-1} \nu^{-1}(a)
$$

where $c_{a} \in K^{*}$ is the coefficient of $\omega_{t a}$ in $a^{*} \nu^{-1}(a)$. We must show that the scalar $c_{a}$ is not changed by the deforming element $f$. This reduces to the following. Suppose $\omega_{i}=b_{1} \ldots b_{m}$ where the $b_{i}$ are arrows. If $x_{i}$ is also a monomial in arrows spanning the same socle space, then we must use the relations to express $x_{i}$ as a scalar multiple of $\omega_{i}$. The relations not at the exceptional vertex are the same as for the preprojective algebra $P(\Delta)$. At the exceptional vertex we substitute, say for $\alpha$ the term $-\beta-\gamma-f(\alpha, \beta)$ (where $\alpha=\bar{a}_{0} a_{0}$ and similarly $\beta$ ). But the term in which $\alpha$ is replaced by $f(\alpha, \beta)$ is zero since $f(\alpha, \beta)$ belongs to a higher power of the radical. So it does not effect this process. This shows our claim. 
Note that this implies that $\eta^{f}$ has finite order. Namely, we know that $\eta$ takes any arrow $a$ to $\pm \nu^{-1}(a)$ and fixes the primitive idempotents. So $\eta^{f}$ fixes the idempotents and takes an arrow $a$ to $\pm \nu^{-1}(a)$. Hence some power of $\eta^{f}$ is the identity.

Proposition 3.4. Let $\Lambda=P^{f}(\Delta)$ be a deformed preprojective algebra of a generalized Dynkin type $\Delta$. Then $\Omega_{\Lambda^{e}}^{3}(\Lambda) \cong{ }_{1} \Lambda_{\phi^{-1}}$, as a $\Lambda$ - $\Lambda$-bimodule for an automorphism $\phi$ of $\Lambda$ of finite order. Moreover $\Omega^{3}(S) \cong \nu^{-1} S$ for any simple $\Lambda$-module $S$.

Proof. We may assume $\Delta \in\left\{\mathbb{D}_{n}, \mathbb{E}_{6}, \mathbb{E}_{7}, \mathbb{E}_{8}, \mathbb{L}_{n}\right\}$. We have seen that $\Lambda$ is selfinjective and that it has the same Cartan matrix as $P(\Delta)$. Furthermore, for each vertex of $Q_{P(\Delta)}$ there is a unique minimal relation starting and ending at this vertex, and there are all the defining relations for the algebra. This implies that for each simple module $S$ it is the case that $\Omega^{3}(S) \cong \nu^{-1} S$. Namely, for each vertex $i$ of $Q_{P(\Delta)}$, denote by $S(i)$ the simple $\Lambda$-module associated to $i$, and by $P(i)$ the preprojective cover of $S(i)$. Consider a minimal projective resolution of $S(i)$,

$$
\ldots \rightarrow Q_{2} \rightarrow Q_{1} \rightarrow Q_{0} \rightarrow S(i) \rightarrow 0 .
$$

Then $Q_{0}=P(i)$, and $Q_{1}$ is the direct sum of all $P(j)$ such that there is an arrow from $i$ to $j$. Then $Q_{2}=P(i)$ since we have a unique minimal relation starting at $i$ and ending at $i$. Now by counting the composition factors we see that the kernel of the map $Q_{2} \rightarrow Q_{1}$ is simple and hence it is $S\left(\nu^{-1}(i)\right)$.

Now using $[\mathrm{H},(1.5)]$ we see that the third syzygy of $\Lambda$ as a $\Lambda$ - $\Lambda$-bimodule has the same top factors as ${ }_{1} \Lambda_{\nu^{-1}}$ and the same composition factors. Hence $\Omega_{\Lambda^{e}}^{3}(\Lambda) \cong_{1} \Lambda_{\phi^{-1}}$ for some automorphism $\phi$ of the algebra $\Lambda$ (which on the top composition factors induces the Nakayama permutation). Actually, GSS, (1.4)] also implies that the third syzygy is isomorphic to such a twisted bimodule.

To complete the proof of Proposition 3.4 we must show that there is such $\phi$ of finite order. Applying Lemma 3.3, one modifies the proof for the preprojective algebra $P(\Delta)$ (see [ES1, (2.3)] and [ES2, (2.2)]) and shows that there is a minimal projective resolution of $\Lambda$ as a $\Lambda$ - $\Lambda$-bimodule of the form

$$
0 \longrightarrow{ }_{1} \Lambda_{\phi^{-1}} \longrightarrow P_{2} \stackrel{\tilde{R}}{\longrightarrow} P_{1} \stackrel{\delta}{\longrightarrow} P_{0} \stackrel{u}{\longrightarrow} \Lambda \longrightarrow 0 .
$$

By $[\mathrm{H},(1.5)]$ the projectives in such a resolution are the same as for the undeformed case. The maps $\delta$ and $u$ can be taken as before (see the text following Proposition 2.1). The map $\tilde{R}$ must be a modification of $R$, but only on $e \otimes e$ where $e$ is the exceptional vertex, to take the deformed relation into account. That is, $\tilde{R}=R+R^{f}$ where $R^{f}$ is defined as follows. Set $R^{f}\left(e_{i} \otimes e_{i}\right)=0$ unless $e_{i}$ is the exceptional vertex. For each path $p=c w_{1} w_{2} \ldots w_{k}$ with $w_{i}$ arrows appearing in $f$ with scalar coefficient $0 \neq c$ define $\rho(p)$ to be

$$
c\left[\left(e \otimes w_{2} w_{3} \ldots w_{k}\right)+\left(w_{1} \otimes w_{3} w_{4} \ldots w_{k}\right)+\ldots+\left(w_{1} w_{2} \ldots w_{k-1} \otimes e\right)\right]
$$

and take $R^{f}(e \otimes e)=\sum \rho(p)$. Now we identify the twisted bimodule with the kernel of the map $\tilde{R}$. Since it is a twisted bimodule isomorphic to ${ }_{1} \Lambda_{\phi^{-1}}$ there are generators $\mu_{i}$, one for each vertex $i$ of $Q_{P(\Delta)}$, which play the role of $e_{i}$ in ${ }_{1} \Lambda_{\phi^{-1}}$, and the twist means that we have

$$
x \mu_{i}=\mu_{i} \phi(x), \quad x \in \Lambda e_{i} .
$$


We will use this to show that $\phi$ has finite order. We have seen that $\mu_{i}$ belongs to $\oplus_{j} e_{i} \Lambda e_{j} \otimes e_{j} \Lambda e_{\nu^{-1}(i)}$ and therefore

$$
\phi\left(e_{i}\right)=e_{\nu^{-1}(i)} .
$$

We take the usual monomial basis $e_{i} \mathcal{B}$ for $e_{i} \Lambda$ as described after Theorem 2.2. Then the standard properties of tensor products over $K$ show that we can write

$$
\mu_{i}=\sum_{b \in e_{i} \mathcal{B}}(-1)^{\operatorname{deg}(b)} b \otimes \check{b}
$$

where $\check{b}$ is then uniquely determined by $b$, and for $b \in \mathcal{B} e_{j}$ the element $\check{b}$ belongs to $e_{j} \Lambda e_{\nu^{-1}(i)}$. We call $\check{b}$ the 'coefficient' of $b$ in $\mu_{i}$. We chose to keep the sign since in the undeformed case, one can take $\mu_{i}=\xi_{i}$ where $\xi_{i}$ was defined before.

Since $\Lambda \mu_{i} \cong \Lambda e_{i}$ as left $\Lambda$-modules we know that the coefficient of $e_{i}$ in $(*)$ is non-zero.

(1) We claim that $\check{e_{i}}$ is a non-zero element in the socle of $e_{i} \Lambda$. By our choice we have

$$
0=\widetilde{R}\left(\mu_{i}\right)=\sum_{b}(-1)^{\operatorname{deg}(b)} b \widetilde{R}\left(e_{t b} \otimes e_{t b}\right) \check{b} .
$$

We calculate the coefficient of $e_{i}$ in the expression on the right hand side. Recall that $\widetilde{R}$ is explicitly known. The only time some element of the form $e_{i} \otimes v$ occurs is within

$$
e_{i} \widetilde{R}\left(e_{i} \otimes e_{i}\right) \check{e}_{i} .
$$

So the coefficient of $e_{i}$ in $\widetilde{R}\left(\mu_{i}\right)$ is equal to

$$
\sum_{t a=i} a \check{e},
$$

and this must be zero. The arrows $a$ ending at $e_{i}$ all start at distinct vertices. So if we multiply $(* *)$ by the appropriate idempotents from the left we deduce $a \check{e}_{i}=0$ for all such arrows. This shows that $\check{e}_{i}$ lies in the socle of $e_{i} \Lambda$. It must be non-zero by the above remark, otherwise $\Lambda \mu_{i}$ would not be isomorphic to $\Lambda e_{i}$.

(2) This shows that we may replace $\mu_{i}$ by a non-zero scalar multiple, and we now normalize it so that we have

$$
\check{e}_{i}=\omega_{i}
$$

which is the fixed monomial in the monomial basis spanning the socle of $e_{i} \Lambda$.

(3) Suppose $a$ is an arrow. We claim that then $\check{a}$ is the unique monomial in the basis which lies in the second socle and satisfies $a \check{a}=\omega_{i}$ (where $a$ starts at vertex $i$ ). Fix such an arrow $a$. Write

$$
\mu_{i}=e_{i} \otimes \omega_{i}-a \otimes \check{a} \pm \ldots
$$

and apply $R$, say $a$ ends at vertex $j$. We have

$$
0=\widetilde{R}\left(\mu_{i}\right)=e_{i} \widetilde{R}\left(e_{i} \otimes e_{i}\right) \omega_{i}-a \widetilde{R}\left(e_{j} \otimes e_{j}\right) \check{a} \pm \ldots
$$

The coefficient of $a$ in this expression is zero. Now $a$ only occurs in the two terms we have written and nowhere else. Assume first that $j$ is not exceptional; then the coefficient is equal to

$$
\omega_{i}-\sum_{t} a_{t} \check{a}=0
$$


where the $a_{t}$ are the arrows ending in $j$. Multiplying with idempotents from the left again gives that for $a_{t} \neq a$ we have $a_{t} \check{a}=0$ and moreover

$$
\omega_{i}=a \check{a} .
$$

This implies the claim. Now consider the case when $j$ is exceptional. If $a$ does not occur in a path of $f$, then we get the same as before, $\omega_{i}=a \check{a}$ and some zero conditions. The zero conditions involve some polynomials, but we get the same conclusion using the grading by powers of the radical. In case $a$ occurs in a path of $f$ then we get zero conditions just with one arrow, but then we get

$$
\omega_{i}=a(1+\psi) \check{a}
$$

where $\psi$ lies in the radical and then again by the grading condition (or by calculation with a basis) we conclude $\omega_{i}=a \check{a}$. For example, in type $\mathbb{D}_{n}$ and $a=a_{0}$ write $f=\alpha f_{1}+\beta f_{2}$ where $f_{1}$ and $f_{2}$ are polynomials in $\alpha=\bar{a}_{0} a_{0}$ and $\beta=\bar{a}_{1} a_{1}$; then $\psi=f_{1}$.

Now we can complete the proof of Proposition 3.4. We now consider $\phi\left(a_{i}\right)$. We know for general reasons that it is of the form

$$
\phi\left(a_{i}\right)=c_{i} a_{i}+\zeta_{i}
$$

for some $c_{i} \in K^{*}$ and $\zeta_{i} \in \operatorname{rad}^{2} \Lambda$. We want to show that $c_{i}= \pm 1$ for all $i$. This will then directly imply that $\phi$ has finite order. Namely, some power of $\phi$ will take any arrow $a$ to $a+z_{a}$ for some $z_{a}$ in the square of the radical. Then this power minus the identity is nilpotent.

We have $a_{i} \mu_{i+1}=\mu_{i} \phi\left(a_{i}\right)$. Now, using $(*)$ we have

$$
a_{i} \mu_{i+1}=\sum_{b \in e_{i+1} \mathcal{B}} \pm a_{i} b \otimes \check{b}=\left[\sum_{d \in e_{i} \mathcal{B}} \pm d \otimes \check{d}\right]\left(c_{i} a_{i}+\zeta_{i}\right) .
$$

We equate the coefficient of $a_{i}$ on both sides (ignoring signs). On the left side this is precisely $\omega_{i+1}$. On the right side it is equal to $\breve{a_{i}}\left(c a_{i}+\zeta_{i}\right)$. These must be equal. Now $\check{a}_{i}$ lies in the second socle, so $\check{a}_{i} \zeta_{i}=0$. On the other hand, using (3) and direct calculation we have $\check{a}_{i} a_{i}= \pm \omega_{i+1}$ and hence $c_{i}= \pm 1$.

The following corollary completes the proof of Theorem 1.1.

Corollary 3.5. Let $\Lambda=P^{f}(\Delta)$ be a deformed preprojective algebra of a generalized Dynkin type $\Delta$. Then there exists a positive integer $m=m_{\Lambda}$ such that $\Omega^{3 m}(M) \cong$ $M$ for any non-projective indecomposable finite-dimensional right $\Lambda$-module $M$.

Proof. In the notation of Proposition 3.4, let $m=m_{\Lambda}$ be the order of $\phi$. Then for any non-projective indecomposable finite-dimensional right $\Lambda$-module $M$, we have $\Omega^{3 m} M \cong M \otimes_{\Lambda} \Omega_{\Lambda^{e}}^{3 m}(\Lambda) \cong M \otimes_{\Lambda} \Lambda \cong M$.

We end this section with an example showing that Corollary 2.6 also holds for some deformed preprojective algebras of generalized Dynkin type.

Example 3.6. Consider a deformed preprojective algebra $\Lambda=P^{f}\left(\mathbb{D}_{4}\right)$ of type $\mathbb{D}_{4}$ with $f$ non-zero. Any such algebra $\Lambda$ is a socle deformation of the preprojective algebra $P\left(\mathbb{D}_{4}\right)$ since the square of the radical of the local algebra at the exceptional vertex is already in the socle. It was proved in [BS3, (5.10)] that there is, up to isomorphism, exactly one self-injective algebra socle equivalent but not isomorphic to $P\left(\mathbb{D}_{4}\right)$, and then necessarily the field $K$ has characteristic 2 . One can take 
$f=x y$ to get this algebra. It is a tame symmetric algebra whose stable AuslanderReiten quiver is isomorphic to the stable Auslander-Reiten quiver of $P\left(\mathbb{D}_{4}\right)$, and hence consists only of stable tubes of ranks 1 and 3 (see [BS2, Theorem 1]). This implies that $\Omega_{\Lambda^{e}}^{3}(\Lambda) \cong \Lambda$. In particular, for any non-projective indecomposable finite-dimensional right $\Lambda$-module $M$, we have $\Omega^{3}(M) \cong M \cong \nu^{-1} M$.

\section{The Gabriel quivers, Cartan matrices and Nakayama permutations}

Given a finite connected graph $\Delta$, a function $\mathrm{d}: \Delta_{0} \rightarrow \mathbb{N}$ on the set $\Delta_{0}$ of vertices of $\Delta$ is called additive (respectively, subadditive) if for any vertex $i \in \Delta_{0}$ we have

$$
2 \mathrm{~d}(i)=\sum_{i-j} \mathrm{~d}(j) \quad\left(\text { respectively, } 2 \mathrm{~d}(i) \geq \sum_{i-j} \mathrm{~d}(j)\right)
$$

where the sum runs over all edges $i-j$ in $\Delta$. Then we have the following fact established in [HPR1] (see also [HPR2]).

Proposition 4.1. Let $\Delta$ be a finite connected graph with no multiple edges. Then $\Delta$ admits a subadditive function which is not additive if and only if $\Delta$ is one of the generalized Dynkin graphs $\mathbb{A}_{n}, \mathbb{D}_{n}, \mathbb{E}_{6}, \mathbb{E}_{7}, \mathbb{E}_{8}$, or $\mathbb{L}_{n}$.

The first aim of this section is to prove the following fact.

Proposition 4.2. Let $\Lambda$ be a basic, connected, finite-dimensional self-injective algebra such that all non-projective simple right $\Lambda$-modules $S$ satisfy $\Omega^{3} S \cong \nu^{-1} S$. Then the Gabriel quiver $Q_{\Lambda}$ of $\Lambda$ is isomorphic to the Gabriel quiver of one of the preprojective algebras of generalized Dynkin type.

Proof. Since $\Lambda$ is basic, $\Lambda \cong K Q / I$, where $Q=Q_{\Lambda}$ and $I$ is an admissible ideal in the path algebra $K Q$ of $Q$. Moreover, $Q_{\Lambda}$ is connected, because $\Lambda$ is connected. We may assume $\Lambda=K Q / I$. Denote by $Q_{0}$ and $Q_{1}$ the sets of vertices and arrows of $Q$, respectively. For each vertex $i \in Q_{0}$, denote by $S(i)$ the simple $\Lambda$-module associated to $i$, and by $P(i)$ the projective cover of $S(i)$. It follows from our assumption that $\Omega^{3} S(i) \cong \nu^{-1} S(i)=S\left(\nu^{-1}(i)\right)$, for any $i \in Q_{0}$. Hence, for every $i \in Q_{0}$, there exists an exact sequence of modules

$$
0 \longrightarrow S\left(\nu^{-1}(i)\right) \longrightarrow P(i) \longrightarrow Q(i) \longrightarrow P(i) \longrightarrow S(i) \longrightarrow 0
$$

where $Q(i)$ is the projective cover of $\operatorname{rad} P(i)$ and the injective envelope of

$$
P(i) / \operatorname{soc} P(i)=P(i) / S\left(\nu^{-1}(i)\right) \text {. }
$$

Hence,

$$
Q(i) \cong \bigoplus_{i \rightarrow j} P(j)
$$

where the sum runs over all arrows $i \rightarrow j$ in $Q$ with the starting vertex $i$. Moreover, we have

$$
\bigoplus_{i \rightarrow j} S\left(\nu^{-1}(j)\right)=\operatorname{soc}\left(\bigoplus_{i \rightarrow j} P(j)\right)=\operatorname{soc} P(i) / S\left(\nu^{-1}(i)\right) .
$$

Hence, for any arrow $i \rightarrow j$ in $Q$ there is exactly one arrow $\nu^{-1}(j) \rightarrow \nu^{-1}(i)$, and consequently exactly one arrow $j \rightarrow i$. Therefore, for any arrow $a$ of $Q$ there exists exactly one arrow $\bar{a}$ such that $i \bar{a}=t a$ and $t \bar{a}=i a$, and moreover $a=\bar{a}$ if and only if $a$ is a loop. Thus we may define the finite connected graph $\Delta$ by replacing in the quiver $Q=Q_{\Lambda}$ each pair $\{a, \bar{a}\}$ of arrows by an undirected edge. Then the set $\Delta_{0}$ 
of vertices of $\Delta$ coincides with $Q_{0}$. Consider the function $\mathrm{d}: \Delta_{0} \rightarrow \mathbb{N}$ defined by $\mathrm{d}(i)=\operatorname{dim}_{K} P(i)$, for $i \in \Delta_{0}$. From the exact sequence

$$
0 \longrightarrow P(i) / S\left(\nu^{-1}(i)\right) \longrightarrow Q(i) \longrightarrow \operatorname{rad} P(i) \longrightarrow 0
$$

we obtain the equality

$$
2(\mathrm{~d}(i)-1)=\sum_{i \rightarrow j} \mathrm{~d}(j)
$$

This implies the inequality

$$
2 \mathrm{~d}(i)>\sum_{i-j} \mathrm{~d}(j)
$$

where the sum runs through all edges $i-j$ of the graph $\Delta$. Therefore, $\mathrm{d}$ is a subadditive but not additive function on $\Delta$. Now applying Proposition 4.1 we obtain that $\Delta$ is one of the generalized Dynkin graphs $\mathbb{A}_{n}, \mathbb{D}_{n}, \mathbb{E}_{6}, \mathbb{E}_{7}, \mathbb{E}_{8}$, or $\mathbb{L}_{n}$. Clearly, $Q=Q_{\Lambda}$ is the Gabriel quiver of the preprojective algebra $P(\Delta)$.

Proposition 4.3. Let $\Lambda$ be a basic, connected, finite-dimensional self-injective algebra such that all non-projective simple right $\Lambda$-modules $S$ satisfy $\Omega^{3} S \cong \nu^{-1} S$. Then there is a generalized Dynkin graph $\Delta$ such that the Cartan matrix $C_{\Lambda}$ of $\Lambda$ coincides with the Cartan matrix $C_{P(\Delta)}$ of $P(\Delta)$, and the Nakayama permutation of $\Lambda$ is the same as the Nakayama permutation of $P(\Delta)$.

Proof. It follows from Proposition 4.2 that $\Lambda \cong K Q / I$, where $Q=Q_{P(\Delta)}$ for a generalized Dynkin graph $\Delta$ and $I$ is an admissible ideal of the path algebra $K Q$. We use the notation from the proof of Proposition 4.2, and identify a $\Lambda$-module with the corresponding representation of the bound quiver $(Q, I)$. For each vertex $i$ of $Q$, let $p(i)=\underline{\operatorname{dim}} P(i)$ and $\epsilon(i)=\underline{\operatorname{dim}} S(i)$ be the dimension-vectors of the projective module $P(i)$ and the simple module $S(i)$ at $i$. Recall that the Cartan matrix $C_{\Lambda}$ of $\Lambda$ is the square matrix whose rows are the dimension-vectors $p(i)$, $i \in Q_{0}$, of the indecomposable projective modules. Denote by $\nu$ the Nakayama permutation of the vertices of $Q$. From our assumption on $\Lambda$, we have the exact sequences

$$
0 \longrightarrow P(i) / S\left(\nu^{-1}(i)\right) \longrightarrow \bigoplus_{i \rightarrow j} P(j) \longrightarrow \operatorname{rad} P(i) \longrightarrow 0,
$$

and consequently the equalities

$$
2 p(i) \epsilon(i)-\epsilon\left(\nu^{-1}(i)\right)=\sum_{i \rightarrow j} p(j),
$$

for all $i \in Q_{0}$. We write this in the matrix form, as

$$
C C_{\Lambda}=E \text {, }
$$

where $E=\left(\epsilon(i)-\epsilon\left(\nu^{-1}(i)\right)\right)_{i \in Q_{0}}$ and $C$ is the Cartan matrix associated to $\Delta$. This is known to be non-singular. Hence, in each case, given $\nu$ there is a unique solution $p(i) \in \mathbb{Q}^{Q_{0}}, i \in Q_{0}$, satisfying the above equation. If $\nu$ is the same as for the preprojective algebra $P(\Delta)$, then we know that the dimension-vectors $p(i)=\underline{\operatorname{dim}} P(i), i \in Q_{0}$, for $\Lambda=P(\Delta)$ satisfy the equation. Therefore, by the uniqueness, the claim follows in this case. In order to finish the proof, we show that for any other possible $\nu$ the solution does not lead to an algebra whose Gabriel quiver is $Q=Q_{P(\Delta)}$. We have few cases to consider. 
(1) Type $\mathbb{A}_{n}$. We may assume $n \geq 2$. Suppose that $\nu$ is the identity. Then the equalities $\left(^{*}\right)$ are of the forms

$$
\begin{aligned}
2 p(0)-p(1) & =2 \epsilon(0), \\
2 p(n-1)-p(n-2) & =2 \epsilon(n-1), \\
\text { and } 2 p(i)-p(i-1)-p(i+1) & =2 \epsilon(i), \text { for } i=1, \ldots, n-2 .
\end{aligned}
$$

Adding all equations we get

$$
p(0)+p(n-1)=2(\epsilon(0)+\epsilon(1)+\cdots+\epsilon(n-2)+\epsilon(n-1))=(2,2, \ldots, 2) .
$$

Since $0=\nu(0)$, the simple module $S(0)$ occurs at least twice as a simple composition factor of the indecomposable projective-injective module $P(0)$. Similarly, $S(n-1)$ occurs at least twice as a simple composition factor of $P(n-1)$. In particular, we obtain $p(0)=(2, \ldots, 0)$. Then we get inductively that $p(1), \ldots, p(n-2)$ all have the last coordinate 0 . Therefore, it follows that there is no arrow in $Q$ from $n-2$ to $n-1$, a contradiction.

(2) Type $\mathbb{D}_{n}$. Suppose that $\nu(0)=1, \nu(1)=0$, and $\nu(i)=i$ for all $i$ with $2 \leq i \leq n-1$. We want to show that then $n$ is odd. The equalities $(*)$ are of the forms

$$
\begin{aligned}
2 p(0)-p(2)=\epsilon(0)+\epsilon(1) & =2 p(1)-p(2), \\
2 p(n-1)-p(n-2) & =2 \epsilon(n-1) \\
2 p(2)-p(0)-p(1)-p(3) & =2 \epsilon(2), \\
\text { and } 2 p(i)-p(i-1)-p(i+1) & =2 \epsilon(i), \text { for } i=3, \ldots, n-2 .
\end{aligned}
$$

In particular, $p(0)=p(1)$ and $p(2)$ have the first two coordinates odd. Further, adding all equations we get

$$
p(0)+p(1)+p(n-1)=p(2)+(2,2, \ldots, 2) .
$$

Since $p(0)=p(1)$, we then conclude that $p(n-1)$ has the first two coordinates odd. Moreover, $p(3)=2 p(2)-2 p(0)-2 \epsilon(2)$ implies that $p(3)_{0}$ is even. Inductively, we deduce that $p(2 k)_{0}$ is odd and $p(2 k+1)_{0}$ is even. Combining these we infer that $n-1$ is even, and so $n$ is odd.

Assume now that $\nu$ is the identity. We claim that then $n$ is even. The equalities $(*)$ are of the forms

$$
\begin{aligned}
2(p(0)-\epsilon(0))=p(2) & =2(p(1)-\epsilon(1)), \\
2(p(n-1)-\epsilon(n-1)) & =p(n-2), \\
2(p(2)-\epsilon(2)) & =p(0)+p(1)+p(3), \\
\text { and } 2(p(i)-\epsilon(i)) & =p(i-1)+p(i+1), \text { for } i=3, \ldots, n-2 .
\end{aligned}
$$

We determine the parity of the first components $p(k)_{0}$ of $p(k)$. Let $N=p(1)_{0}$. Then $2(p(0)-\epsilon(0))=2(p(1)-\epsilon(1))$ implies that $p(0)_{0}=N+1$. Further, $p(2)=$ $2(p(1)-\epsilon(1))$ implies $p(2)_{0}=2 N$, and hence is even. Finally, from

$$
p(3)=2(p(2)-\epsilon(2))-p(0)-p(1),
$$

we obtain $p(3)_{0}=4 N-(N+1)-N=2 N-1$, so is odd. Inductively, we then deduce that

$$
p(2 k)_{0} \text { is even and } p(2 k+1)_{0} \text { is odd. }
$$


Adding all equations $(*)$ gives

$$
\begin{aligned}
p(0)+p(1)+p(n-1) & =p(2)+2\left(\sum_{0 \leq i \leq n-1} \epsilon(i)\right) \\
& =p(0)+p(1)-\epsilon(0)-\epsilon(1)+2\left(\sum_{0 \leq i \leq n-1} \epsilon(i)\right),
\end{aligned}
$$

and hence $p(n-1)=(1,1,2,2, \ldots, 2)$. Therefore, $p(n-1)_{0}$ is odd and hence $n-1$ is odd. Thus $n$ is even.

Assume $\Delta=\mathbb{D}_{4}$ and $\nu(1)=0, \nu(3)=1, \nu(0)=3, \nu(2)=2$. Then the equalities $\left(^{*}\right)$ are of the form

$$
\begin{gathered}
2 p(0)-\epsilon(0)-\epsilon(1)=p(2) \\
2 p(1)-\epsilon(1)-\epsilon(3)=p(2) \\
2 p(2)-2 \epsilon(2)=p(0)+p(1)+p(3), 2 p(3)-\epsilon(3)-\epsilon(1)=p(2) .
\end{gathered}
$$

In particular, we get

$$
\begin{aligned}
3 p(2) & =2(p(0)+p(1)+p(3)-\epsilon(0)-\epsilon(1)-\epsilon(3)) \\
& =4 p(2)-2(2 \epsilon(2)+\epsilon(0)+\epsilon(1)+\epsilon(3)) .
\end{aligned}
$$

Hence, $p(2)=2(2 \epsilon(2)+\epsilon(0)+\epsilon(1)+\epsilon(3))$. But then we have $2 p(0)=3 \epsilon(0)+$ $3 \epsilon(1)+4 \epsilon(2)+2 \epsilon(3)$, a contradiction.

(3) Type $\mathbb{E}_{6}$. Suppose $\nu$ is the identity. Then the equalities $\left(^{*}\right)$ are of the form

$$
\begin{aligned}
& 2(p(0)-\epsilon(0))=p(3), \\
& 2(p(1)-\epsilon(1))=p(2), \\
& 2(p(2)-\epsilon(2))=p(1)+p(3), \\
& 2(p(3)-\epsilon(3))=p(0)+p(2)+p(4), \\
& 2(p(4)-\epsilon(4))=p(3)+p(5), \\
& 2(p(5)-\epsilon(5))=p(4) .
\end{aligned}
$$

Let $N=p(1)_{1}$. Then $p(2)_{1}=2(N-1)$ and $p(3)_{1}=3 N-4$. Moreover, the equalities $p(4)_{1}=2 p(5)_{1}$ and $2 p(4)_{1}=p(3)_{1}+p(5)_{1}$ imply $3 p(5)_{1}=3 N-4$, a contradiction.

(4) Types $\mathbb{E}_{7}, \mathbb{E}_{8}, \mathbb{L}_{n}$. The unique Nakayama permutation is the identity.

\section{Proof of Theorem 1.2}

Let $\Lambda$ be a basic, connected, finite-dimensional self-injective algebra such that all non-projective simple right $\Lambda$-modules $S$ satisfy $\Omega^{3} S \cong \nu^{-1} S$. We know from Proposition 4.2 that the Gabriel quiver $Q=Q_{\Lambda}$ of $\Lambda$ coincides with the Gabriel quiver $Q_{P(\Delta)}$ of the preprojective algebra $P(\Delta)$ of a generalized Dynkin type $\Delta \in$ $\left\{\mathbb{A}_{n}, \mathbb{D}_{n}, \mathbb{E}_{6}, \mathbb{E}_{7}, \mathbb{E}_{8}, \mathbb{L}_{n}\right\}$. Moreover, by Proposition 4.3, the Cartan matrices and the Nakayama permutations of $\Lambda$ and $P(\Delta)$ are the same. We will prove that $\Lambda$ is isomorphic to a deformed preprojective algebra $P^{f}(\Delta)$, for an admissible element $f$ of the local algebra $R(\Delta)$, and hence (ii) implies (i). We may assume that $\Lambda$ is non-simple, and hence $Q_{\Lambda}$ has at least one arrow.

Let $I$ be an admissible ideal in the path algebra $K Q$ of $Q=Q_{\Lambda}$ such that $\Lambda \cong K Q / I$. We may assume that $\Lambda=K Q / I$. For each vertex $i$ of $Q$, let $e_{i}$ 
be the primitive idempotent corresponding to $i$ and $P(i)=e_{i} \Lambda$ the associated indecomposable projective $\Lambda$-module. We will also identify an arrow of $Q$ with its coset modulo $I$. Also denote by $\nu^{-1}$ the inverse Nakayama permutation of the vertices of $Q$, that is, $e_{\nu^{-1}(i)} \Lambda$ is the projective cover of the socle of $e_{i} \Lambda$, for any vertex $i$ of $Q$.

Lemma 5.1. The indecomposable projective $\Lambda$-modules have the same Loewy length.

Proof. It follows from our assumption on $\Lambda$ that, for each vertex $i$ of $Q$, there exists an exact sequence of $\Lambda$-modules

$$
0 \longrightarrow P(i) / \operatorname{soc} P(i) \longrightarrow \bigoplus_{i \rightarrow j} P(j) \longrightarrow \operatorname{rad} P(i) \longrightarrow 0
$$

where the middle term is the projective cover of $\operatorname{rad} P(i)$ and the injective envelope of $P(i) / \operatorname{soc} P(i)$. Hence we obtain the inequalities $L L(P(j)) \geq L L(P(i))$, for all arrows $i \rightarrow j$ in $Q$. Since in the quiver $Q=Q_{\Delta}$ there are paths with any prescribed starting and ending vertices, we conclude that $L L(P(l))=L L(P(k))$ for all vertices $l$ and $k$ of $Q$.

Lemma 5.2. For each vertex $i$ of $Q$, there are invertible elements $u_{a} \in e_{i} \Lambda e_{i}$, for all arrows a of $Q$ with $i a=i$, such that

$$
\sum_{a, i a=i} a \bar{a} u_{a}=0
$$

Proof. Fix a vertex $i$ of $Q$. We have an exact sequence of $\Lambda$-modules

$$
0 \longrightarrow e_{i} \Lambda / \operatorname{soc}\left(e_{i} \Lambda\right) \stackrel{w_{i}}{\longrightarrow} \bigoplus_{a, i a=i} e_{t a} \Lambda \stackrel{\pi_{i}}{\longrightarrow} e_{i}(\operatorname{rad} \Lambda) \longrightarrow 0
$$

where the middle term is the projective cover of $e_{i}(\operatorname{rad} \Lambda)=\operatorname{rad}\left(e_{i} \Lambda\right)$ and the injective envelope of $e_{i} \Lambda / \operatorname{soc}\left(e_{i} \Lambda\right)$. Observe also that

$$
e_{i}(\operatorname{rad} \Lambda)=\sum_{a, i a=i} a \Lambda
$$

Hence, we may assume that the restriction $\pi_{a}: e_{t a} \Lambda \rightarrow e_{i}(\operatorname{rad} \Lambda)$ of $\pi_{i}$ to $e_{t a} \Lambda$ assigns to the primitive idempotent $e_{t a}$ the arrow $a$. Furthermore, we note that $\operatorname{soc}\left(e_{i} \Lambda / \operatorname{soc}\left(e_{i} \Lambda\right)\right)$ is a direct sum of pairwise non-isomorphic simple modules, because it is isomorphic to the socle of $\bigoplus_{a, i a=i} e_{t a} \Lambda$. Denote by $w_{a}: e_{i} \Lambda / \operatorname{soc}\left(e_{i} \Lambda\right) \rightarrow$ $e_{t a} \Lambda$ the composition of $w_{i}$ with the projection of $\bigoplus_{a, i a=i} e_{t a} \Lambda$ on $e_{t a} \Lambda$. Then, for each arrow $a$ of $Q$ with $i a=i, w_{a}$ has non-zero restriction to the direct summand of $\operatorname{soc}\left(e_{i} \Lambda / \operatorname{soc}\left(e_{i} \Lambda\right)\right)$ isomorphic to $\operatorname{soc}\left(e_{t a} \Lambda\right)$. Moreover, it follows from Lemma 5.1 that $L L\left(e_{i} \Lambda / \operatorname{soc}\left(e_{i} \Lambda\right)\right)=L L\left(\operatorname{rad} e_{t a} \Lambda\right)$, for any arrow $a$ of $Q$ with $i a=i$. Observe that there is a non-zero path $a b$ in $\Lambda$ from $i$ to $\nu^{-1}(t a)$ such that $\bar{a} a b$ generates the socle of $e_{t a} \Lambda$ and the coset $a b+\operatorname{soc}\left(e_{i} \Lambda\right)$ generates the direct summand of $\operatorname{soc}\left(e_{i} \Lambda / \operatorname{soc}\left(e_{i} \Lambda\right)\right)$ isomorphic to $\operatorname{soc}\left(e_{t a} \Lambda\right)$. This implies that $L L(a b \Lambda)=L L\left(\operatorname{rad}\left(e_{t a} \Lambda\right)\right)$ and $w_{a}\left(e_{i}+\operatorname{soc}\left(e_{i} \Lambda\right)\right) \in e_{t a}(\operatorname{rad} \Lambda) e_{i} \backslash e_{t a}(\operatorname{rad} \Lambda)^{2} e_{i}$. Moreover, since $e_{t a}(\operatorname{rad} \Lambda) e_{i} / e_{t a}(\operatorname{rad} \Lambda)^{2} e_{i}$ is the one-dimensional space generated by $\bar{a}+e_{t a}(\operatorname{rad} \Lambda)^{2} e_{i}$, we conclude that $w_{a}\left(e_{i}+\operatorname{soc}\left(e_{i} \Lambda\right)\right)=\bar{a} u_{a}$ for an invertible element $u_{a} \in e_{i} \Lambda e_{i}$. Hence we infer that the monomorphism $w_{i}$ is given by

$$
w_{i}\left(e_{i}+\operatorname{soc}\left(e_{i} \Lambda\right)\right)=\sum_{a, i a=i} \bar{a} u_{a} \in \bigoplus_{a, i a=i} e_{t a}(\operatorname{rad} \Lambda) e_{i} .
$$


Therefore, we obtain the required relation

$$
0=\pi_{i} w_{i}\left(e_{i}+\operatorname{soc}\left(e_{i} \Lambda\right)\right)=\sum_{a, i a=i} a \bar{a} u_{a} .
$$

Lemma 5.3. For each vertex $i$ of $Q$, there is exactly one minimal relation in $e_{i} I e_{i}$.

Proof. Fix a vertex $i$ of $Q$. We know from Lemma 5.2 that $e_{i} I e_{i} \neq 0$. Let $\varrho$ be a non-zero element of $e_{i} I e_{i}$. Then $\varrho$ is a linear combination of paths in $Q$ from $i$ to $i$. Observe that every path from $i$ to $i$ is of the form $a b$ for an arrow $a$ with $i a=i$ and a path $b$ from $t a$ to $i$. Hence, $\varrho$ is of the form

$$
\varrho=\sum_{a, i a=i} a x_{a}
$$

where $x_{a} \in e_{t a}(K Q)_{e_{i}}$. Clearly, $\varrho=0$ in $\Lambda$. Consider the monomorphism $\varphi: e_{i} \Lambda \rightarrow$ $\bigoplus_{a, i a=i} e_{t a} \Lambda$ such that $\varphi\left(e_{i}\right)=\sum_{a, i a=i} x_{a}$, and the canonical exact sequence of $\Lambda$ modules

$$
0 \longrightarrow e_{i} \Lambda / \operatorname{soc}\left(e_{i} \Lambda\right) \stackrel{w_{i}}{\longrightarrow} \bigoplus_{a, i a=i} e_{t a} \Lambda \stackrel{\pi_{i}}{\longrightarrow} e_{i}(\operatorname{rad} \Lambda) \longrightarrow 0 .
$$

Then $\pi_{i} \varphi=0$, and hence there exists a $\Lambda$-homomorphism $\psi: e_{i} \Lambda \rightarrow e_{i} \Lambda / \operatorname{soc}\left(e_{i} \Lambda\right)$ such that $\varphi=w_{i} \psi$. Moreover, $\psi$ is given by $\psi\left(e_{i}\right)=\mu+\operatorname{soc}\left(e_{i} \Lambda\right)$ for some $\mu \in e_{i} \Lambda e_{i}$. Then, in the notation from the proof of Lemma 5.2. we have

$$
\varphi\left(e_{i}\right)=w_{i} \psi\left(e_{i}\right)=\sum_{a, i a=i}\left(\bar{a} u_{a}\right) \mu
$$

for some invertible elements $u_{a} \in e_{i} \Lambda e_{i}$. Therefore, we obtain

$$
\varrho=\sum_{a, i a=i} a x_{a}=\left(\sum_{a, i a=i} a \bar{a} u_{a}\right) \mu .
$$

The following proposition proves the necessity part of Theorem 1.2 .

Proposition 5.4. $\Lambda$ is isomorphic to $P^{f}(\Delta)$ for some admissible element $f$ of $R(\Delta)$.

Proof. We know that $\Lambda=K Q / I$, where $Q=Q_{P(\Delta)}$ for a generalized Dynkin graph $\Delta$ with at least one edge and, for each vertex $i$ of $Q$, there is exactly one minimal relation in $e_{i} I e_{i}$, described by Lemma 5.2. We will first prove that $\Lambda$ admits a bound quiver presentation $\Lambda \cong K Q / J$, where $J$ is an admissible ideal of $K Q$ containing the relations

$$
\sum_{a, i a=v} a \bar{a},
$$

for all ordinary vertices $v$ of $Q$. Let

$$
r \underset{a_{r}}{\stackrel{a_{r}}{\rightleftarrows}} r+1
$$


be a subquiver of $Q$ with $r$ being the end vertex of $Q$. Then, by Lemma 5.2, we have a relation $a_{r} \bar{a}_{r} u_{r}=0$, for an invertible element $u_{r} \in e_{r} \Lambda e_{r}$. Then, multiplying by $u_{r}^{-1}$, we get $a_{r} \bar{a}_{r}=0$. Further, let

$$
i \underset{a_{i}}{\stackrel{a_{i}}{\rightleftarrows}} i+1 \underset{\bar{a}_{i+1}}{\stackrel{a_{i+1}}{\rightleftarrows}} \cdot \ldots \cdot \underset{\bar{a}_{r-2}}{\stackrel{a_{r-2}}{\rightleftarrows}} r-1 \underset{\bar{a}_{r-1}}{\stackrel{a_{r-1}}{\rightleftarrows}} r \underset{a_{r}}{\stackrel{a_{r}}{\rightleftarrows}} r+1
$$

be a subquiver of $Q$, with $i, i+1, \ldots, r$ being the ordinary vertices of $Q$ and $i$ the end vertex of $Q$, and assume that

$$
a_{i} \bar{a}_{i}=0, \bar{a}_{i} a_{i}+a_{i+1} \bar{a}_{i+1}=0, \ldots, \bar{a}_{r-2} a_{r-2}+a_{r-1} \bar{a}_{r-1}=0 .
$$

Applying Lemma 5.2. we conclude that there are invertible elements $u_{r-1}, u_{r} \in$ $e_{r} \Lambda e_{r}$ such that

$$
\bar{a}_{r-1} a_{r-1} u_{r-1}+a_{r} \bar{a}_{r} u_{r}=0 .
$$

Then we get the relation

$$
\bar{a}_{r-1} a_{r-1}+a_{r} \bar{a}_{r}\left(u_{r} u_{r-1}^{-1}\right)=0 .
$$

Since $u_{r} u_{r-1}^{-1}$ is invertible in $e_{r} \Lambda e_{r}$, we have $\bar{b}_{r}=\bar{a}_{r}\left(u_{r} u_{r-1}^{-1}\right) \in e_{r+1}(\operatorname{rad} \Lambda) e_{r} \backslash$ $e_{r+1}(\operatorname{rad} \Lambda)^{2} e_{r}$. Hence, replacing $\bar{a}_{r}$ by $\bar{b}_{r}$, we obtain a new bound quiver presentation of $\Lambda$ such that

$$
\bar{a}_{r-1} a_{r-1}+a_{r} \bar{b}_{r}=0,
$$

and the relations $(*)$ are unchanged. Therefore, we may assume that $\Lambda \cong K Q / J$, where $J$ contains the relations $\sum_{a, i a=v} a \bar{a}$, for all ordinary vertices $v$ of $Q$. We will now analyse the relation at the exceptional vertex of $Q$, given by Lemma 5.2 .

(1) Assume $\Delta=\mathbb{A}_{n}$. Then, since by our assumption $n \geq 2$, at the exceptional vertex 0 of $Q$ we have a relation $a_{0} \bar{a}_{0} u_{0}=0$ for an invertible element $u_{0} \in e_{0} \Lambda e_{0}$. Hence, multiplying by $u_{0}^{-1}$, we obtain the required relation $a_{0} \bar{a}_{0}=0$. Therefore, $\Lambda$ is isomorphic to $P(\Delta)$.

(2) Assume $\Delta=\mathbb{D}_{n}$. Then, at the exceptional vertex 2, we have, by Lemma 5.2 , a relation of the form

$$
\bar{a}_{0} a_{0} u_{0}+\bar{a}_{1} a_{1} u_{1}+a_{2} \bar{a}_{2} u_{2}=0,
$$

for invertible elements $u_{0}, u_{1}, u_{2} \in e_{2} \Lambda e_{2}$. Multiplying by $u_{2}^{-1}$, we get the relation

$$
\bar{a}_{0} a_{0} v_{0}+\bar{a}_{1} a_{1} \bar{v}_{1}+a_{2} \bar{a}_{2}=0,
$$

with $v_{0}=u_{0} u_{2}^{-1}$ and $v_{1}=u_{1} u_{2}^{-1}$. Moreover, we may write $v_{0}=\lambda e_{2}+x_{0}$ and $v_{1}=\mu e_{2}+x_{1}$ for some $\lambda, \mu \in K \backslash\{0\}$ and $x_{0}, x_{1} \in \operatorname{rad} e_{2} \Lambda e_{2}$. Hence we obtain

$$
\left(\lambda \bar{a}_{0} a_{0}+\mu \bar{a}_{1} a_{1}+a_{2} \bar{a}_{2}\right)+\left(\lambda \bar{a}_{0} a_{0} y_{0}+\mu \bar{a}_{1} a_{1} y_{1}\right)=0,
$$

where $y_{0}=\lambda^{-1} x_{0}$ and $y_{1}=\mu^{-1} x_{1}$. Replacing $\bar{a}_{0}$ by $\bar{b}_{0}=\lambda \bar{a}_{0}$ and $\bar{a}_{1}$ by $\bar{b}_{1}=\mu \bar{a}_{1}$, we get the relation

$$
\bar{b}_{0} a_{0}+\bar{b}_{1} a_{1}+a_{2} \bar{a}_{2}+\left(\bar{b}_{0} a_{0} y_{0}+\bar{b}_{1} a_{1} y_{1}\right)=0 .
$$

Moreover, $a_{0} \bar{b}_{0}=a_{0} \lambda \bar{a}_{0}=\lambda a_{0} \bar{a}_{0}=0$ and $a_{1} \bar{b}_{1}=a_{1} \mu \bar{a}_{1}=\mu a_{1} \bar{a}_{1}=0$. Further, $\bar{b}_{0} a_{0} y_{0}, \bar{b}_{1} a_{1} y_{1} \in e_{2}(\operatorname{rad} \Lambda)^{2} e_{2},\left(\bar{b}_{0} a_{0}\right)^{2}=0,\left(\bar{b}_{1} a_{1}\right)^{2}=0,\left(a_{2} \bar{a}_{2}\right)^{n-2}=0$, and consequently $e_{2} \Lambda e_{2}$ is generated by $\bar{b}_{0} a_{0}$ and $\bar{b}_{1} a_{1}$, and is isomorphic to the local algebra $R(\Delta)=R\left(\mathbb{D}_{n}\right)$. In particular, $\bar{b}_{0} a_{0} y_{0}+\bar{b}_{1} a_{1} y_{1}=f\left(\bar{b}_{0} a_{0}, \bar{b}_{1} a_{1}\right)$ for an element $f \in(\operatorname{rad} R(\Delta))^{2}$. Summing up, we proved that there is an algebra epimorphism $P^{f}(\Delta) \rightarrow \Lambda$. On the other hand, by Proposition 4.3, the Cartan matrix $C_{\Lambda}$ of $\Lambda$ is the same as the Cartan matrix $C_{P(\Delta)}$ of $P(\Delta)$, and hence 
$\operatorname{dim}_{K} \Lambda=\operatorname{dim}_{K} P(\Delta)$. We also know that $\operatorname{dim}_{K} P^{f}(\Delta)=\operatorname{dim}_{K} P(\Delta)$ due to Lemma 3.2. Hence $\operatorname{dim}_{K} \Lambda=\operatorname{dim}_{K} P^{f}(\Delta)$, and so $\Lambda$ is isomorphic to $P^{f}(\Delta)$.

(3) Assume $\Delta=\mathbb{E}_{n}, n=6,7,8$. Applying arguments as in (2), we conclude that, at the exceptional vertex 3 , we have a relation of the form

$$
\bar{b}_{0} a_{0}+\bar{b}_{2} a_{2}+a_{3} \bar{a}_{3}+\left(\bar{b}_{0} a_{0} y_{0}+\bar{b}_{2} a_{2} y_{2}\right)=0,
$$

where $y_{0}, y_{2} \in e_{3}(\operatorname{rad} \Lambda) e_{3}$ and $\bar{b}_{0}=\lambda \bar{a}_{0}, \bar{b}_{2}=\mu \bar{a}_{2}$, for some $\lambda, \mu \in K \backslash\{0\}$. Then again $a_{0} \bar{b}_{0}=a_{0} \lambda \bar{a}_{0}=a_{0} \bar{a}_{0} \lambda=0$. Moreover, for $\bar{b}_{1}=\lambda \bar{a}_{1}$, the relations $a_{1} \bar{a}_{1}=0$ and $\bar{a}_{1} a_{1}+a_{2} \bar{a}_{2}=0$ imply the relations $a_{1} \bar{b}_{1}=0$ and $\bar{b}_{1} a_{1}+a_{2} \bar{b}_{2}=0$. Further, we have $\left(\bar{b}_{0} a_{0}\right)^{2}=0,\left(\bar{b}_{2} a_{2}\right)^{3}=0$ and $\left(a_{3} \bar{a}_{3}\right)^{n-3}=0$. Since $\bar{b}_{0} a_{0} y_{0}, \bar{b}_{2} a_{2} y_{2} \in$ $e_{3}(\operatorname{rad} \Lambda)^{2} e_{3}$, we conclude that $e_{3} \Lambda e_{3}$ is generated by $\bar{b}_{0} a_{0}$ and $\bar{b}_{2} a_{2}$, and is isomorphic to the local algebra $R(\Delta)=R\left(\mathbb{E}_{n}\right)$. In particular, $\bar{b}_{0} a_{0} y_{0}+\bar{b}_{2} a_{2} y_{2}=$ $f\left(\bar{b}_{0} a_{0}, \bar{b}_{2} a_{2}\right)$ for an element $f \in(\operatorname{rad} R(\Delta))^{2}$. Therefore, we deduce that there is an algebra epimorphism $P^{f}(\Delta) \rightarrow \Lambda$. Now invoking Lemma 3.2 and Proposition 4.3. we conclude that $\operatorname{dim}_{K} \Lambda=\operatorname{dim}_{K} P(\Delta)=\operatorname{dim}_{K} P^{f}(\Delta)$, and consequently that $\Lambda$ is isomorphic to $P^{f}(\Delta)$.

(4) Assume $\Delta=\mathbb{L}_{n}$. Applying arguments as above, we infer that, at the exceptional vertex 0 , we have a relation of the form

$$
\varepsilon^{2} \lambda+a_{0} \bar{a}_{0}+\varepsilon^{2} x=0,
$$

for $\lambda \in K \backslash\{0\}$ and $x \in e_{0}(\operatorname{rad} \Lambda) e_{0}$. Then, for a square root $\mu$ of $\lambda$ and $\varepsilon_{0}=\mu \varepsilon$, we obtain the relation

$$
\varepsilon_{0}^{2}+a_{0} \bar{a}_{0}+\varepsilon_{0}^{2} x=0 .
$$

Further, $\left(a_{0} \bar{a}_{0}\right)^{n}=0$ implies $\varepsilon_{0}^{2 n}=0$, and hence the local algebra $e_{0} \Lambda e_{0}$ is generated by $\varepsilon_{0}$, and is isomorphic to $R(\Delta)=R\left(\mathbb{L}_{n}\right)$. Moreover, $\varepsilon_{0}^{2} x=\varepsilon_{0} f\left(\varepsilon_{0}\right)$ for an element $f \in(\operatorname{rad} R(\Delta))^{2}$. Therefore, we proved that there is an algebra epimorphism $P^{f}(\Delta) \rightarrow \Lambda$. Applying again Lemma 3.2 and Proposition 4.3, we conclude that $\operatorname{dim}_{K} \Lambda=\operatorname{dim}_{K} P(\Delta)=\operatorname{dim}_{K} P^{f}(\Delta)$, and consequently $\Lambda$ is isomorphic to $P^{f}(\Delta)$.

\section{Proof of Theorem 1.3}

Throughout this section we assume that $K$ is an algebraically closed field of characteristic 2 . For each generalized Dynkin graph $\Delta$ different from $\mathbb{A}_{n}$ and $\mathbb{L}_{1}$, we will exhibit a deformed preprojective algebra $P^{f}(\Delta)$ over $K$ which is not isomorphic to the preprojective algebra $P(\Delta)$. In fact, we will show that for the generalized Dynkin graphs $\Delta \in\left\{\mathbb{D}_{n}, \mathbb{L}_{n}\right\}$ the number of pairwise non-isomorphic deformed preprojective algebras of type $\Delta$ grows with $n$.

Let $\Delta=\mathbb{L}_{n}$ with $n \geq 2$. For each element $f \in \operatorname{rad}^{2} R\left(\mathbb{L}_{n}\right)$ of the form $f=$ $\left(\lambda_{1} x^{2}+\lambda_{2} x^{3}+\cdots+\lambda_{2 n-3} x^{2 n-1}\right)+\left(x^{2 n}\right)$, with $\lambda_{1}, \lambda_{2}, \ldots, \lambda_{2 n-3} \in K$, we put

$$
L_{n}\left(\lambda_{1}, \ldots, \lambda_{2 n-3}\right)=P^{f}\left(\mathbb{L}_{n}\right) .
$$

Proposition 6.1. For each $k \in\{1, \ldots, n-1\}, \lambda_{2 k-1} \in K \backslash\{0\}$, and $\lambda_{2 k+1}, \lambda_{2 k+2}$, $\ldots, \lambda_{2 n-3} \in K$, we have

$$
L_{n}\left(0,0, \ldots, 0, \lambda_{2 k-1}, 0,0, \ldots, 0\right) \supsetneqq L_{n}\left(0,0, \ldots, 0, \lambda_{2 k+1}, \lambda_{2 k+2}, \ldots, \lambda_{2 n-3}\right) .
$$

In particular, $L_{n}(1,0,0, \ldots, 0), L_{n}(0,0,1,0,0, \ldots, 0), \ldots, L_{n}(0,0, \ldots, 0,1,0,0)$, $L_{n}(0,0, \ldots, 0,1), L_{n}(0,0, \ldots, 0)=P\left(\mathbb{L}_{n}\right)$ form a family of pairwise non-isomorphic deformed preprojective algebras of type $\mathbb{L}_{n}$. 
Proof. Let $\lambda_{2 k-1} \in K \backslash\{0\}$, and $\lambda_{2 k+1}, \lambda_{2 k+2}, \ldots, \lambda_{2 n-3} \in K$, for some $k \in$ $\{1, \ldots, n-1\}$. Suppose there exists an algebra isomorphism

$$
\varphi: L_{n}\left(0,0, \ldots, 0, \lambda_{2 k-1}, 0,0, \ldots, 0\right) \rightarrow L_{n}\left(0,0, \ldots, 0, \lambda_{2 k+1}, \lambda_{2 k+2}, \ldots, \lambda_{2 n-3}\right) .
$$

Then $\varphi$ is determined by the elements of the form

$$
\begin{aligned}
\varphi\left(a_{l}\right) & =\sum_{i=0}^{n-2-l}\left(\alpha_{i}^{(l)}\left(a_{l} \bar{a}_{l}\right)^{i} a_{l}+\alpha_{i+n-1-l}^{(l)}\left(a_{l} \bar{a}_{l}\right)^{i} \bar{a}_{l-1} \ldots \bar{a}_{0} \varepsilon a_{0} \ldots a_{l}\right), \\
\varphi\left(\bar{a}_{l}\right) & =\sum_{i=0}^{n-2-l}\left(\bar{\alpha}_{i}^{(l)} \bar{a}_{l}\left(a_{l} \bar{a}_{l}\right)^{i}+\bar{\alpha}_{i+n-1-l}^{(l)} \bar{a}_{l} \ldots \bar{a}_{0} \varepsilon a_{0} \ldots a_{l-1}\left(a_{l} \bar{a}_{l}\right)^{i}\right), \\
\varphi(\varepsilon) & =\sum_{i=0}^{2 n-2} \gamma_{i} \varepsilon^{i+1},
\end{aligned}
$$

for $l \in\{0, \ldots, n-2\}$ and parameters $\gamma_{i}, \alpha_{i}^{(l)}, \bar{\alpha}_{i}^{(l)} \in K$ with $\gamma_{0}, \alpha_{0}^{(l)}, \bar{\alpha}_{0}^{(l)}$ non-zero.

In the following computations we are especially interested in coefficients corresponding to the non-zero paths of length $2 k+1$.

From the relations of

$$
L_{n}\left(0,0, \ldots, 0, \lambda_{2 k-1}, 0,0, \ldots, 0\right)
$$

and

$$
L_{n}\left(0, \ldots, 0, \lambda_{2 k+1}, \lambda_{2 k+2}, \ldots, \lambda_{2 n-3}\right)
$$

corresponding to the vertex 0 we obtain

$$
\begin{aligned}
0 & =\varphi\left(a_{0} \bar{a}_{0}+\varepsilon^{2}+\lambda_{2 k-1} \varepsilon^{2 k+1}\right) \\
& =\cdots+\left(\lambda_{2 k-1} \gamma_{0}^{2 k+1}+\sum_{i=0}^{k-1}\left(\alpha_{i}^{(0)} \bar{\alpha}_{n+k-2-i}^{(0)}+\bar{\alpha}_{i}^{(0)} \alpha_{n+k-2-i}^{(0)}\right)\right) \varepsilon^{2 k+1}+\ldots,
\end{aligned}
$$

because

$$
\sum_{i=0}^{2 k-1} \gamma_{i} \gamma_{2 k-1-i} \varepsilon^{2 k+1}=2 \sum_{i=0}^{k-1} \gamma_{i} \gamma_{2 k-1-i} \varepsilon^{2 k+1}=0
$$

Hence we get $\lambda_{2 k-1} \gamma_{0}^{2 k+1}=\sum_{i=0}^{k-1}\left(\alpha_{i}^{(0)} \bar{\alpha}_{n+k-2-i}^{(0)}+\bar{\alpha}_{i}^{(0)} \alpha_{n+k-2-i}^{(0)}\right)$.

From the relations corresponding to the vertex 1 we obtain

$$
\sum_{i=0}^{k-1}\left(\alpha_{i}^{(0)} \bar{\alpha}_{n+k-2-i}^{(0)}+\bar{\alpha}_{i}^{(0)} \alpha_{n+k-2-i}^{(0)}\right)=\sum_{i=0}^{k-2}\left(\alpha_{i}^{(1)} \bar{\alpha}_{n+k-2-i}^{(1)}+\bar{\alpha}_{i}^{(1)} \alpha_{n+k-2-i}^{(1)}\right),
$$

and so on up to the vertex $k$, in which we obtain

$$
\alpha_{0}^{(k-1)} \bar{\alpha}_{n-k}^{(k-1)}+\bar{\alpha}_{0}^{(k-1)} \alpha_{n-k}^{(k-1)}=0
$$

Summing up we obtain

$$
\begin{aligned}
\lambda_{2 k-1} \gamma_{0}^{2 k+1} & =\sum_{i=0}^{k-1}\left(\alpha_{i}^{(0)} \bar{\alpha}_{n+k-2-i}^{(0)}+\bar{\alpha}_{i}^{(0)} \alpha_{n+k-2-i}^{(0)}\right) \\
& =\cdots=\alpha_{0}^{(k-1)} \bar{\alpha}_{n-k}^{(k-1)}+\bar{\alpha}_{0}^{(k-1)} \alpha_{n-k}^{(k-1)}=0 .
\end{aligned}
$$

This contradicts $\gamma_{0} \neq 0 \neq \lambda_{2 k-1}$.

Let $\Delta=\mathbb{D}_{n}$ with $n \geq 4$. Consider the monomials $v_{k} \in K\langle x, y\rangle, 0 \leq k<\left[\frac{n-2}{2}\right]$, defined as follows:

$$
v_{k}(x, y)=\left\{\begin{array}{cl}
(x y)^{k / 2} & \text { if } k \text { even } \\
(x y)^{(k-1) / 2} x & \text { if } k \text { odd }
\end{array}\right.
$$


For $\underline{\lambda}=\left(\lambda_{1}, \lambda_{2}, \ldots, \lambda_{2 n-7}\right) \in K^{2 n-7}$ denote by $f(\underline{\lambda})$ the element of $\operatorname{rad}^{2} R\left(\mathbb{D}_{n}\right)$ of the form

$$
f(\underline{\lambda})=\left(\sum_{k=1}^{n-3} \lambda_{2 k-1} v_{k+1}(x, y)+\sum_{k=1}^{n-4} \lambda_{2 k} v_{k+1}(y, x)\right)+\left(x^{2}, y^{2},(x+y)^{n-2}\right),
$$

and set

$$
D_{n}\left(\lambda_{1}, \lambda_{2}, \ldots, \lambda_{2 n-7}\right)=P^{f(\underline{\lambda})}\left(\mathbb{D}_{n}\right)
$$

Proposition 6.2. For each $k \in\left\{0, \ldots,\left[\frac{n-2}{2}\right]\right\}, \lambda_{4 k+1} \in K \backslash\{0\}$, and $\lambda_{4 k+5}, \lambda_{4 k+6}$, $\ldots, \lambda_{2 n-7} \in K$, we have

$$
D_{n}\left(0,0, \ldots, 0, \lambda_{4 k+1}, 0,0, \ldots, 0\right) \supsetneqq D_{n}\left(0,0, \ldots, 0, \lambda_{4 k+5}, \lambda_{4 k+6}, \ldots, \lambda_{2 n-7}\right) .
$$

In particular, $D_{n}(1,0, \ldots, 0), D_{n}(0,0,0,0,1,0, \ldots, 0), \ldots, D_{n}(0, \ldots, 0,1)$ for $n$ even (respectively, $D_{n}(0, \ldots, 0,1,0,0)$ for $n$ odd), and $D_{n}(0,0, \ldots, 0)=P\left(\mathbb{D}_{n}\right)$ form a family of pairwise non-isomorphic deformed preprojective algebras of type $\mathbb{D}_{n}$.

Proof. Let $\lambda_{4 k+1} \in K \backslash\{0\}$ and $\lambda_{4 k+5} \lambda_{4 k+6}, \ldots, \lambda_{2 n-7} \in K$, for some $k \in\{0, \ldots$, $\left.\left[\frac{n-2}{2}\right]\right\}$, set

$$
\begin{aligned}
& u_{0, i}=v_{i}\left(\bar{a}_{0} a_{0}, \bar{a}_{1} a_{1}\right), \quad u_{1, i}=v_{i}\left(\bar{a}_{1} a_{1}, \bar{a}_{0} a_{0}\right), \\
& u_{0, k}^{\prime}=\left\{\begin{array}{cl}
\left(\bar{a}_{1} a_{1} \bar{a}_{0} a_{0}\right)^{k / 2} & \text { if } k \text { even }, \\
\left(\bar{a}_{0} a_{0} \bar{a}_{1} a_{1}\right)^{(k-1) / 2} \bar{a}_{0} a_{0} & \text { if } k \text { odd },
\end{array}\right. \\
& u_{1, k}^{\prime}=\left\{\begin{array}{cc}
\left(\bar{a}_{0} a_{0} \bar{a}_{1} a_{1}\right)^{k / 2} & \text { if } k \text { even, } \\
\left(\bar{a}_{1} a_{1} \bar{a}_{0} a_{0}\right)^{(k-1) / 2} \bar{a}_{1} a_{1} & \text { if } k \text { odd } .
\end{array}\right.
\end{aligned}
$$

Suppose

$$
\varphi: D_{n}\left(0,0, \ldots, 0, \lambda_{4 k+1}, 0,0, \ldots, 0\right) \rightarrow D_{n}\left(0,0, \ldots, 0, \lambda_{4 k+5}, \lambda_{4 k+6}, \ldots, \lambda_{2 n-7}\right)
$$

is an algebra isomorphism. Then $\varphi$ is determined by the elements of the form

$$
\begin{gathered}
\varphi\left(a_{0}\right)=\sum_{i=0}^{n-3} \alpha_{i}^{(0)} a_{0} u_{1, i}, \quad \varphi\left(\bar{a}_{0}\right)=\sum_{i=0}^{n-3} \bar{\alpha}_{i}^{(0)} u_{1, i}^{\prime} \bar{a}_{0}, \\
\varphi\left(a_{1}\right)=\sum_{i=0}^{n-3} \alpha_{i}^{(1)} a_{1} u_{0, i}, \quad \varphi\left(\bar{a}_{1}\right)=\sum_{i=0}^{n-3} \bar{\alpha}_{i}^{(1)} u_{0, i}^{\prime} \bar{a}_{1}, \\
\varphi\left(a_{l}\right)=\sum_{i=0}^{n-2-l}\left(\alpha_{i}^{(l)}\left(a_{l} \bar{a}_{l}\right)^{i} a_{l}+\beta_{i}^{(l)} \bar{a}_{l-1} \bar{a}_{l-2} \ldots \bar{a}_{2} \bar{a}_{1} a_{1} a_{2} \ldots a_{l-1}\left(a_{l} \bar{a}_{l}\right)^{i} a_{l}\right), \\
\varphi\left(\bar{a}_{l}\right)=\sum_{i=0}^{n-2-l}\left(\bar{\alpha}_{i}^{(l)}\left(\bar{a}_{l} a_{l}\right)^{i} \bar{a}_{l}+\bar{\beta}_{i}^{(l)}\left(\bar{a}_{l} a_{l}\right)^{i} \bar{a}_{l} \bar{a}_{l-1} \bar{a}_{l-2} \ldots \bar{a}_{2} \bar{a}_{1} a_{1} a_{2} \ldots a_{l-1}\right),
\end{gathered}
$$

for $l \in\{2, \ldots, n-2\}$ and some parameters $\alpha_{i}^{(l)}, \bar{\alpha}_{i}^{(l)}, \beta_{i}^{(l)}, \bar{\beta}_{i}^{(l)} \in K$, with $\alpha_{0}^{(l)}, \bar{\alpha}_{0}^{(l)}, \beta_{0}^{(l)}$, $\bar{\beta}_{0}^{(l)}$ non-zero, $0 \leq l \leq n-2$. Clearly, $\alpha_{0}^{(0)} \bar{\alpha}_{0}^{(0)}=\cdots=\alpha_{0}^{(n-2)} \bar{\alpha}_{0}^{(n-2)}$.

We will compute the coefficients corresponding to the paths of length $4(k+1)$ in $\varphi\left(a_{l} \bar{a}_{l}\right)$ and $\varphi\left(\bar{a}_{l} a_{l}\right)$, for $l=0, \ldots, n-2$.

Invoking the relations at the vertices 0 and 1 , we obtain

$$
\varphi\left(a_{0} \bar{a}_{0}\right)=\cdots+\sum_{i=0}^{2 k+1} \alpha_{i}^{(0)} \bar{\alpha}_{2 k+1-i}^{(0)} a_{0} u_{1,2 k+1} \bar{a}_{0}+\ldots
$$


and

$$
\varphi\left(a_{1} \bar{a}_{1}\right)=\cdots+\sum_{i=0}^{2 k+1} \alpha_{i}^{(1)} \bar{\alpha}_{2 k+1-i}^{(1)} a_{1} u_{0,2 k+1} \bar{a}_{1}+\ldots,
$$

respectively. Hence $\sum_{i=0}^{2 k+1} \alpha_{i}^{(0)} \bar{\alpha}_{2 k+1-i}^{(0)}=\sum_{i=0}^{2 k+1} \alpha_{i}^{(1)} \bar{\alpha}_{2 k+1-i}^{(1)}=0$.

For $l=3, \ldots, 2 k+3$, we obtain

$$
\begin{gathered}
\varphi\left(\bar{a}_{l-1} a_{l-1}\right)=\cdots+\sum_{i=0}^{2 k+1} \alpha_{i}^{(l-1)} \bar{\alpha}_{2 k+1-i}^{(l-1)}\left(\bar{a}_{l-1} a_{l-1}\right)^{2 k+2} \\
+\sum_{i=0}^{2 k+3-l}\left(\alpha_{i}^{(l-1)} \bar{\beta}_{2 k+3-l-i}^{(l-1)}+\beta_{i}^{(l-1)} \bar{\alpha}_{2 k+3-l-i}^{(l-1)}\right) \\
\cdot \bar{a}_{l-1} \ldots \bar{a}_{1} a_{1} \ldots a_{l-1}\left(\bar{a}_{l-1} a_{l-1}\right)^{2 k+3-l}+\ldots
\end{gathered}
$$

Similarly, for $l=3, \ldots, 2 k+2$, we obtain

$$
\begin{gathered}
\varphi\left(a_{l} \bar{a}_{l}\right)=\cdots+\sum_{i=0}^{2 k+1} \alpha_{i}^{(l)} \bar{\alpha}_{2 k+1-i}^{(l)}\left(a_{l} \bar{a}_{l}\right)^{2 k+2} \\
+\sum_{i=0}^{2 k+2-l}\left(\alpha_{i}^{(l)} \bar{\beta}_{2 k+2-l-i}^{(l)}+\beta_{i}^{(l)} \bar{\alpha}_{2 k+2-l-i}^{(l)}\right)\left(a_{l} \bar{a}_{l}\right)^{2 k+2-l} \bar{a}_{l-1} \ldots \bar{a}_{1} a_{1} \ldots \bar{a}_{l-1}+\ldots
\end{gathered}
$$

Hence, invoking the relation at the vertex $l$, for $l=3, \ldots, 2 k+2$, we obtain

$$
\begin{aligned}
\sum_{i=0}^{2 k+3-l} & \left(\alpha_{i}^{(l-1)} \bar{\beta}_{2 k+3-l-i}^{(l-1)}+\beta_{i}^{(l-1)} \bar{\alpha}_{2 k+3-l-i}^{(l-1)}\right) \\
& =\sum_{i=0}^{2 k+2-l}\left(\alpha_{i}^{(l)} \bar{\beta}_{2 k+2-l-i}^{(l)}+\beta_{i}^{(l)} \bar{\alpha}_{2 k+2-l-i}^{(l)}\right)
\end{aligned}
$$

and, using the relation at the vertex $2 k+3$, we get

$$
\alpha_{0}^{(2 k+2)} \bar{\beta}_{0}^{(2 k+2)}+\beta_{0}^{(2 k+2)} \bar{\alpha}_{0}^{(2 k+2)}=0 .
$$

Summing up, we obtain

$$
\sum_{i=0}^{2 k} \alpha_{i}^{(2)} \bar{\beta}_{2 k-i}^{(2)}+\sum_{i=0}^{2 k} \beta_{i}^{(2)} \bar{\alpha}_{2 k-i}^{(2)}=\cdots=0 .
$$

Further, we have

$$
\begin{aligned}
\varphi\left(a_{2} \bar{a}_{2}\right)=\ldots & +\sum_{i=0}^{2 k+1} \alpha_{i}^{(2)} \bar{\alpha}_{2 k+1-i}^{(2)}\left(a_{2} \bar{a}_{2}\right)^{2 k+2} \\
& +\sum_{i=0}^{2 k} \alpha_{i}^{(2)} \bar{\beta}_{2 k+1-i}^{(2)}\left(a_{2} \bar{a}_{2}\right)^{2 k+1} \bar{a}_{1} a_{1} \\
& +\sum_{i=0}^{2 k} \beta_{i}^{(2)} \bar{\alpha}_{2 k+1-i}^{(2)} \bar{a}_{1} a_{1}\left(a_{2} \bar{a}_{2}\right)^{2 k+1}+\ldots \\
=\ldots & +\left(\sum_{i=0}^{2 k+1} \alpha_{i}^{(2)} \bar{\alpha}_{2 k+1-i}^{(2)}+\sum_{i=0}^{2 k} \alpha_{i}^{(2)} \bar{\beta}_{2 k-i}^{(2)}\right) u_{0,2 k+2} \\
& +\left(\sum_{i=0}^{2 k+1} \alpha_{i}^{(2)} \bar{\alpha}_{2 k+1-i}^{(2)}+\sum_{i=0}^{2 k} \beta_{i}^{(2)} \bar{\alpha}_{2 k-i}^{(2)}\right) u_{1,2 k+2}+\ldots
\end{aligned}
$$

Hence, the coefficients of $u_{0,2 k+2}$ and $u_{1,2 k+2}$ in $\varphi\left(\bar{a}_{2} a_{2}\right)$ are equal. Further, it follows from $\sum_{i=0}^{2 k+1} \alpha_{i}^{(0)} \bar{\alpha}_{2 k+1-i}^{(0)}=0$ (respectively, from $\sum_{i=0}^{2 k+1} \alpha_{i}^{(1)} \bar{\alpha}_{2 k+1-i}^{(1)}=0$ ) 
that the coefficients of $u_{0,2 k+2}$ and $u_{1,2 k+2}$ in $\varphi\left(\bar{a}_{0} a_{0}\right)$ (respectively, in $\varphi\left(\bar{a}_{1} a_{1}\right)$ ) are also equal. Indeed, we have

$$
\varphi\left(\bar{a}_{0} a_{0}\right)=\cdots+\sum_{i=0}^{k} \alpha_{2 i+1}^{(0)} \bar{\alpha}_{2 k-2 i}^{(0)} u_{0,2 k+2}+\sum_{i=0}^{k} \alpha_{2 i}^{(0)} \bar{\alpha}_{2 k+1-2 i}^{(0)} u_{1,2 k+2}+\ldots
$$

and

$$
\varphi\left(\bar{a}_{1} a_{1}\right)=\cdots+\sum_{i=0}^{k} \alpha_{2 i}^{(1)} \bar{\alpha}_{2 k+1-2 i}^{(1)} u_{0,2 k+2}+\sum_{i=0}^{k} \alpha_{2 i+1}^{(1)} \bar{\alpha}_{2 k-2 i}^{(1)} u_{1,2 k+2}+\ldots
$$

On the other hand, $\varphi\left(\lambda_{4 k+1} u_{0,2 k+2}\right)=\lambda_{4 k+1}\left(\alpha_{0} \bar{\alpha}_{0}\right)^{2 k+2} u_{0,2 k+2}+\ldots$

Finally, invoking the relation at the vertex 2 , we obtain

$$
\begin{aligned}
0 & =\varphi(0)=\varphi\left(a_{0} \bar{a}_{0}+a_{1} \bar{a}_{1}+\bar{a}_{2} a_{2}+\lambda_{4 k+1} u_{0,2 k+2}\right) \\
& =\cdots+\lambda_{4 k+1}\left(\alpha_{0} \bar{\alpha}_{0}\right)^{2 k+2} u_{0,2 k+2}+\mu\left(u_{0,2 k+2}+u_{1,2 k+2}\right)+\ldots
\end{aligned}
$$

for some $\mu \in K$. Hence, $\alpha_{0} \bar{\alpha}_{0}=\mu=0$ or $\lambda_{4 k+1}=\mu=0$, a contradiction.

Let $\Delta=\mathbb{E}_{n}, 6 \leq n \leq 8$. For $\lambda, \mu \in K$ denote by $f(\lambda, \mu)$ the element of $\operatorname{rad}^{2} R\left(\mathbb{E}_{n}\right)$ of the form

$$
f(\lambda, \mu)=\lambda x y+\mu y x+\left(x^{2}, y^{3},(x+y)^{n-3}\right),
$$

and put

$$
E_{n}(\lambda, \mu)=P^{f(\lambda, \mu)}\left(\mathbb{E}_{n}\right) .
$$

Proposition 6.3. Let $\lambda, \mu \in K$, with $\lambda \neq \mu$. Then $E_{n}(\lambda, \mu)$ is not isomorphic to the preprojective algebra $P\left(\mathbb{E}_{n}\right)=E_{n}(0,0)$.

Proof. Suppose that $\varphi: E_{n}(0,0) \rightarrow E_{n}(\lambda, \mu)$ is an algebra isomorphism. Then $\varphi$ is determined by the elements of the form

$$
\begin{aligned}
\varphi\left(a_{0}\right) & =\alpha_{0}^{(0)} a_{0}+\alpha_{1}^{(0)} a_{0} \bar{a}_{2} a_{2}+\ldots, \\
\varphi\left(\bar{a}_{0}\right) & =\bar{\alpha}_{0}^{(0)} \bar{a}_{0}+\bar{\alpha}_{1}^{(0)} \bar{a}_{2} a_{2} \bar{a}_{0}+\ldots, \\
\varphi\left(a_{1}\right) & =\alpha_{0}^{(1)} a_{1}+\ldots, \\
\varphi\left(\bar{a}_{1}\right) & =\bar{\alpha}_{0}^{(1)} \bar{a}_{1}+\ldots, \\
\varphi\left(a_{2}\right) & =\alpha_{0}^{(2)} a_{2}+\alpha_{1}^{(2)} a_{2} a_{3} \bar{a}_{3}+\alpha_{2}^{(2)} a_{2} \bar{a}_{2} a_{2}+\ldots, \\
\varphi\left(\bar{a}_{2}\right) & =\bar{\alpha}_{0}^{(2)} \bar{a}_{2}+\bar{\alpha}_{1}^{(2)} a_{3} \bar{a}_{3} \bar{a}_{2}+\bar{\alpha}_{2}^{(2)} \bar{a}_{2} a_{2} \bar{a}_{2}+\ldots, \\
\varphi\left(a_{3}\right) & =\alpha_{0}^{(3)} a_{3}+\alpha_{1}^{(3)} \bar{a}_{2} a_{2} a_{3}+\alpha_{2}^{(3)} a_{3} \bar{a}_{3} a_{3}+\ldots, \\
\varphi\left(\bar{a}_{3}\right) & =\bar{\alpha}_{0}^{(3)} \bar{a}_{3}+\bar{\alpha}_{1}^{(3)} \bar{a}_{3} \bar{a}_{2} a_{2}+\bar{\alpha}_{2}^{(3)} \bar{a}_{3} a_{3} \bar{a}_{3}+\ldots, \\
\varphi\left(a_{i}\right) & =\alpha_{0}^{(i)} a_{i}+\alpha_{1}^{(i)} \bar{a}_{i-1} a_{i-1} a_{i}+\ldots, \quad \text { for } 3<i<n-2, \\
\varphi\left(\bar{a}_{i}\right) & =\bar{\alpha}_{0}^{(i)} \bar{a}_{i}+\bar{\alpha}_{1}^{(i)} \bar{a}_{i} \bar{a}_{i-1} a_{i-1}+\ldots, \quad \text { for } 3<i<n-2, \\
\varphi\left(a_{n-2}\right) & =\alpha_{0}^{(n-2)} a_{n-2}+\ldots, \\
\varphi\left(\bar{a}_{n-2}\right) & =\bar{\alpha}_{0}^{(n-2)} \bar{a}_{n-2}+\ldots
\end{aligned}
$$

for some parameters $\alpha_{i}^{(l)}, \bar{\alpha}_{i}^{(l)} \in K$, with $\alpha_{0}^{(l)}, \bar{\alpha}_{0}^{(l)}$ non-zero, $0 \leq l \leq n-2$.

We note that $\alpha_{0}^{(0)} \bar{\alpha}_{0}^{(0)}=\alpha_{0}^{(1)} \bar{\alpha}_{0}^{(1)}=\alpha_{0}^{(2)} \bar{\alpha}_{0}^{(2)}=\alpha_{0}^{(3)} \bar{\alpha}_{0}^{(3)}=\alpha_{0}^{(4)} \bar{\alpha}_{0}^{(4)}=\cdots=$ $\alpha_{0}^{(n-2)} \bar{\alpha}_{0}^{(n-2)}$. 
Denote that $\beta_{i}^{(j)}=\left(\alpha_{i}^{(0)}\right)^{-1} \alpha_{i}^{(j)}$ and $\bar{\beta}_{i}^{(j)}=\left(\bar{\alpha}_{i}^{(0)}\right)^{-1} \bar{\alpha}_{i}^{(j)}$. Invoking the relation at the vertex 0 , we obtain

$$
\begin{aligned}
0 & =\varphi\left(a_{0} \bar{a}_{0}\right) \\
& =\alpha_{0}^{(0)} \bar{\alpha}_{0}^{(0)} a_{0} \bar{a}_{0}+\left(\alpha_{1}^{(0)} \bar{\alpha}_{0}^{(0)}+\alpha_{0}^{(0)} \bar{\alpha}_{1}^{(0)}\right) a_{0} \bar{a}_{2} a_{2} \bar{a}_{0}+\ldots,
\end{aligned}
$$

and thus $\beta_{1}^{(0)}+\bar{\beta}_{1}^{(0)}=0$. Invoking the relation at the vertex 2 , we obtain

$$
\begin{aligned}
0= & \varphi\left(\bar{a}_{1} a_{1}+a_{2} \bar{a}_{2}\right) \\
= & \alpha_{0}^{(1)} \bar{\alpha}_{0}^{(1)} \bar{a}_{1} a_{1}+\alpha_{0}^{(2)} \bar{\alpha}_{0}^{(2)} a_{2} \bar{a}_{2}+\left(\alpha_{1}^{(2)} \bar{\alpha}_{0}^{(2)}+\alpha_{0}^{(2)} \bar{\alpha}_{1}^{(2)}\right) a_{2} a_{3} \bar{a}_{3} \bar{a}_{2} \\
& +\left(\alpha_{2}^{(2)} \bar{\alpha}_{0}^{(2)}+\alpha_{0}^{(2)} \bar{\alpha}_{2}^{(2)}\right) a_{2} \bar{a}_{2} a_{2} \bar{a}_{2}+\ldots,
\end{aligned}
$$

hence $\beta_{1}^{(2)}+\bar{\beta}_{1}^{(2)}=0$ (note that $\left.a_{2} \bar{a}_{2} a_{2} \bar{a}_{2}=\bar{a}_{1} a_{1} \bar{a}_{1} a_{1}=0\right)$. Using the relation at the vertex 4 , we obtain

$$
\begin{aligned}
0= & \varphi\left(\bar{a}_{3} a_{3}+a_{4} \bar{a}_{4}\right) \\
= & \alpha_{0}^{(3)} \bar{\alpha}_{0}^{(3)} \bar{a}_{3} a_{3}+\alpha_{0}^{(4)} \bar{\alpha}_{0}^{(4)} a_{4} \bar{a}_{4}+\left(\alpha_{1}^{(3)} \bar{\alpha}_{0}^{(3)}+\alpha_{0}^{(3)} \bar{\alpha}_{1}^{(3)}\right) \bar{a}_{3} \bar{a}_{2} a_{2} a_{3} \\
& +\left(\alpha_{2}^{(3)} \bar{\alpha}_{0}^{(3)}+\alpha_{0}^{(3)} \bar{\alpha}_{2}^{(3)}+\alpha_{1}^{(4)} \bar{\alpha}_{0}^{(4)}+\alpha_{0}^{(4)} \bar{\alpha}_{1}^{(4)}\right) \bar{a}_{3} a_{3} \bar{a}_{3} a_{3}+\ldots
\end{aligned}
$$

(assuming $\alpha_{1}^{(4)}=\bar{\alpha}_{1}^{(4)}=0$ for $n=6$ ). Note that, for $n=6$ we have $\bar{a}_{3} a_{3} \bar{a}_{3} a_{3}=0$, and for $n=7,8$ the elements $\bar{a}_{3} a_{3} a_{4} \bar{a}_{4}=\bar{a}_{3} a_{3} \bar{a}_{3} a_{3}=a_{4} \bar{a}_{4} \bar{a}_{3} a_{3}$ and $\bar{a}_{3} \bar{a}_{2} a_{2} a_{3}$ are linearly independent. Hence $\beta_{1}^{(3)}+\bar{\beta}_{1}^{(3)}=0$.

Observe also that

$$
\begin{gathered}
a_{3} \bar{a}_{3} a_{3} \bar{a}_{3}=\bar{a}_{2} a_{2} \bar{a}_{2} a_{2}+\bar{a}_{2} a_{2} \bar{a}_{0} a_{0}+\bar{a}_{0} a_{0} \bar{a}_{2} a_{2}+\ldots, \\
a_{3} \bar{a}_{3} \bar{a}_{2} a_{2}=\bar{a}_{2} a_{2} \bar{a}_{2} a_{2}+\bar{a}_{0} a_{0} \bar{a}_{2} a_{2}+\ldots \\
\bar{a}_{2} a_{2} a_{3} \bar{a}_{3}=\bar{a}_{2} a_{2} \bar{a}_{2} a_{2}+\bar{a}_{2} a_{2} \bar{a}_{0} a_{0}+\ldots
\end{gathered}
$$

Finally, invoking the relation at the vertex 3 , we obtain

$$
\begin{aligned}
0= & \varphi\left(\bar{a}_{0} a_{0}+\bar{a}_{2} a_{2}+a_{3} \bar{a}_{3}\right) \\
= & \alpha_{0}^{(0)} \bar{\alpha}_{0}^{(0)} \bar{a}_{0} a_{0}+\alpha_{0}^{(2)} \bar{\alpha}_{0}^{(2)} \bar{a}_{2} a_{2}+\alpha_{0}^{(3)} \bar{\alpha}_{0}^{(3)} a_{3} \bar{a}_{3} \\
& +\alpha_{1}^{(0)} \bar{\alpha}_{0}^{(0)} \bar{a}_{0} a_{0} \bar{a}_{2} a_{2}+\alpha_{0}^{(0)} \bar{\alpha}_{1}^{(0)} \bar{a}_{2} a_{2} \bar{a}_{0} a_{0}+\left(\alpha_{2}^{(2)} \bar{\alpha}_{0}^{(2)}+\alpha_{0}^{(2)} \bar{\alpha}_{2}^{(2)}\right) \bar{a}_{2} a_{2} \bar{a}_{2} a_{2} \\
& +\left(\alpha_{2}^{(3)} \bar{\alpha}_{0}^{(3)}+\alpha_{0}^{(3)} \bar{\alpha}_{2}^{(3)}\right) \bar{a}_{3} a_{3} \bar{a}_{3} a_{3}+\left(\alpha_{1}^{(2)} \bar{\alpha}_{0}^{(2)}+\alpha_{1}^{(3)} \bar{\alpha}_{0}^{(3)}\right) \bar{a}_{2} a_{2} a_{3} \bar{a}_{3} \\
& +\left(\alpha_{0}^{(2)} \bar{\alpha}_{1}^{(2)}+\alpha_{0}^{(3)} \bar{\alpha}_{1}^{(3)}\right) a_{3} \bar{a}_{3} \bar{a}_{2} a_{2}+\ldots \\
= & \alpha_{0}^{(0)} \bar{\alpha}_{0}^{(0)}\left(\bar{a}_{0} a_{0}+\bar{a}_{2} a_{2}+a_{3} \bar{a}_{3}\right. \\
& +\left(\beta_{1}^{(2)}+\bar{\beta}_{1}^{(2)}+\beta_{1}^{(3)}+\bar{\beta}_{1}^{(3)}+\beta_{2}^{(2)}+\bar{\beta}_{2}^{(2)}+\beta_{2}^{(3)}+\bar{\beta}_{2}^{(3)}\right) \bar{a}_{2} a_{2} \bar{a}_{2} a_{2} \\
& +\left(\bar{\beta}_{1}^{(0)}+\beta_{1}^{(2)}+\beta_{1}^{(3)}+\beta_{2}^{(3)}+\bar{\beta}_{2}^{(3)}\right) \bar{a}_{2} a_{2} \bar{a}_{0} a_{0} \\
& +\left(\beta_{1}^{(0)}+\bar{\beta}_{1}^{(2)}+\bar{\beta}_{1}^{(3)}+\beta_{2}^{(3)}+\bar{\beta}_{2}^{(3)}\right) \bar{a}_{0} a_{0} \bar{a}_{2} a_{2} \\
& +\ldots) .
\end{aligned}
$$

Then we have

$$
\lambda=\left(\beta_{1}^{(0)}+\bar{\beta}_{1}^{(2)}+\bar{\beta}_{1}^{(3)}+\beta_{2}^{(3)}+\bar{\beta}_{2}^{(3)}\right)
$$

and

$$
\mu=\left(\bar{\beta}_{1}^{(0)}+\beta_{1}^{(2)}+\beta_{1}^{(3)}+\beta_{2}^{(3)}+\bar{\beta}_{2}^{(3)}\right) .
$$


Summing up,

$$
\lambda+\mu=2\left(\beta_{2}^{(3)}+\bar{\beta}_{2}^{(3)}\right)+\left(\beta_{1}^{(0)}+\bar{\beta}_{1}^{(0)}\right)+\left(\beta_{1}^{(2)}+\bar{\beta}_{1}^{(2)}\right)+\left(\beta_{1}^{(3)}+\bar{\beta}_{1}^{(3)}\right)=0 .
$$

This contradicts our assumption that $K$ is of characteristic 2 and $\mu \neq \lambda$.

\section{REFERENCES}

[A] M. Artin, Maximal orders of global dimension and Krull dimension two, Invent. Math. 84 (1986), 195-222. MR0830045 (88e:16008a)

[AR1] M. Auslander, I. Reiten, Almost split sequences for rational double points, Trans. Amer. Math. Soc. 302 (1987), 87-97. MR0887498 (88e:13011)

[AR2] M. Auslander, I. Reiten, D Tr-periodic modules and functors, in: Representation Theory of Algebras, CMS Conference Proceedings, Vol. 18, Amer. Math. Soc., Providence, RI, 1996, pp. 39-50. MR.1388043 (98g:16008)

[ARS] M. Auslander, I. Reiten, S.O. Smalø, Representation Theory of Artin Algebras, in: Cambridge Studies in Advanced Mathematics, Vol. 36, Cambridge University Press, Cambridge, UK, 1995. MR1314422 (96c:16015)

[BGL] D. Baer, W. Geigle, H. Lenzing, The preprojective algebra of a tame hereditary Artin algebra, Comm. Algebra 15 (1987), 425-457. MR0876985 (88i:16036)

[BS1] J. Białkowski, A. Skowroński, Selfinjective algebras of tubular type, Colloq. Math. 94 (2002), 175-194. MR1967373 (2004a:16019)

[BS2] J. Białkowski, A. Skowroński, On tame weakly symmetric algebras of tubular type, Archiv Math. 81 (2003), 142-154. MR2009556 (2004k:16039)

[BS3] J. Białkowski, A. Skowroński, Socle deformations of selfinjective algebras of tubular type, J. Math. Soc. Japan 56 (2004) 3, 687-716. MR2071668 (2005c:16018)

[B] R.O. Buchweitz, Finite representation type and periodic Hochschild (co-)homology, in: Trends in the Representation Theory of Finite Dimensional Algebras, Contemp. Math., Vol. 229, Amer. Math. Soc., Providence, RI, 1998, pp. 81-109. MR1676212 (2000b:18001)

[CBH] W. Crawley-Boevey, M.P. Holland, Noncommutative deformations of Kleinian singularities, Duke Math. J. 92 (1998), 605-635. MR1620538 (99f:14003)

[DR1] V. Dlab, C.M. Ringel, The preprojective algebra of a modulated graph, in: Representation Theory II, in: Lecture Notes in Math., Vol. 832, Springer-Verlag, Berlin/New York, 1980, pp. 216-231. MR0607155 (83c:16022)

[DR2] V. Dlab, C.M. Ringel, The module theoretical approach to quasi-hereditary algebras, in: Representations of Algebras and Related Topics, in: London Mathematical Society Lecture Note Series, Vol. 168, Cambridge University Press, Cambridge, UK, 1992, pp. 200-224. MR1211481 (94f:16026)

[ES1] K. Erdmann, N. Snashall, On Hochschild cohomology of preprojective algebras I, J. Algebra 205 (1998), 391-412. MR1632808 (99e:16013)

[ES2] K. Erdmann, N. Snashall, Preprojective algebras of Dynkin type, periodicity and the second Hochschild cohomology, in: Algebras and Modules II, CMS Conference Proceedings, Vol. 24, Amer. Math. Soc., Providence, RI, 1998, pp. 183-193. MR.1648626 (99h:16016)

[G] P. Gabriel, Auslander-Reiten sequences and representation-finite algebras, in: Representation Theory I, in: Lecture Notes in Math., Vol. 831, Springer-Verlag, Berlin/New York, 1980, pp. 1-71. MR0607140 (82i:16030)

[GSS] E.L. Green, N. Snashall, O. Solberg, The Hochschild cohomology ring of a selfinjective algebra of finite representation type, Proc. Amer. Math. Soc. 131 (2003) 11, 3387-3393. MR.1990627 (2004c:16013)

[GP] I.M. Gelfand, V.A. Ponomarev, Model algebras and representations of graphs, Funkc. Anal. Priloz. 13 (1979), 1-12. MR0545362 (82a:16030)

$[\mathrm{H}]$ D. Happel, Hochschild cohomology of finite dimensional algebras, in: Séminaire d'Algèbre Paul Dubreil et Marie-Paul Malliavin, in: Lecture Notes in Math., Vol. 1404, Springer-Verlag, Berlin/New York, 1989, pp. 108-126. MR 1035222 (91b:16012)

[HPR1] D. Happel, U. Preiser, C.M. Ringel, Binary polyhedral groups and Euclidean diagrams, Manuscripta Math. 31 (1980), 317-329. MR0576503 (82g:20019) 
[HPR2] D. Happel, U. Preiser, C.M. Ringel, Vinberg's characterization of Dynkin diagrams using subadditive functions with application to D Tr-periodic modules, in: Representation Theory II, in: Lecture Notes in Math., Vol. 832, Springer-Verlag, Berlin/New York, 1980, pp. 280-294. MR0607159 (82g:16027)

[K] P. Kronheimer, The construction of $A L E$ spaces as hyper-Kähler quotients, J. Diff. Geom. 29 (1989), 665-683. MR0992334 (90d:53055)

[L1] G. Lusztig, Quivers, perverse sheaves and quantized enveloping algebras, J. Amer. Math. Soc. 4 (1991), 365-421. MR1088333 (91m:17018)

[L2] G. Lusztig, Affine quivers and canonical bases, Publ. IHES 76 (1992), 111-163.

[Rie] C. Riedtmann, Algebren, Darstellungsköcher und zurück, Comment Math. Helv. 55 (1980), 199-224. MR0576602 (82k:16039)

[Rin1] C.M. Ringel, The preprojective algebra of tame quiver: the irreducible components of the module varieties, in: Trends in the Representation Theory of Finite Dimensional Algebras, Contemp. Math., Vol. 229, Amer. Math. Soc., Providence, RI, 1998, pp. 293306. MR:1676227 (2000b:16028)

[Rin2] C.M. Ringel, The preprojective algebra of a quiver, in: Algebras and Modules II, CMS Conference Proceedings, Vol. 24, Amer. Math. Soc., Providence, RI, 1998, pp. 467-480. MR.1648647 (99i:16031)

[S] A. Schofield, Wild algebras with periodic Auslander-Reiten translate, manuscript.

[Y] K. Yamagata, Frobenius algebras, in: Handbook of algebra, Vol. 1, Amsterdam/New York, Elsevier 1996, pp. 841-887. MR1421820 (97k:16022)

Faculty of Mathematics and Computer Science, Nicolaus Copernicus University, Chopina 12/18, 87-100 Toruń, Poland

E-mail address: jb@mat.uni.torun.pl

Mathematical Institute, University of Oxford, 24-29 St. Giles, Oxford OX1 3LB, UNITED KINGDOM

E-mail address: erdmann@maths.ox.ac.uk

Faculty of Mathematics and Computer Science, Nicolaus Copernicus University, Chopina 12/18, 87-100 Toruń, Poland

E-mail address: skowron@mat.uni.torun.pl 